\title{
Competitive equilibria in a comonotone market
}

\author{
Tim J. Boonen ${ }^{1}(1) \cdot$ Fangda Liu $^{2}\left([) \cdot\right.$ Ruodu Wang $^{2}$ (I)
}

Received: 3 June 2020 / Accepted: 6 October 2020 / Published online: 17 October 2020

(c) The Author(s) 2020

\begin{abstract}
We investigate competitive equilibria in a special type of incomplete markets, referred to as a comonotone market, where agents can only trade such that their risk allocation is comonotonic. The comonotone market is motivated by the no-sabotage condition. For instance, in a standard insurance market, the allocation of risk among the insured, the insurer and the reinsurers is assumed to be comonotonic a priori to the risk-exchange. Two popular classes of preferences in risk management and behavioral economics, dual utilities (DU) and rank-dependent expected utilities (RDU), are used to formulate agents' objectives. We present various results on properties and characterization of competitive equilibria in this framework, and in particular their relation to complete markets. For DU-comonotone markets, we find the equilibrium in closed form and for RDU-comonotone markets, we find the equilibrium in closed form in special cases. The fundamental theorems of welfare economics are established in both the DU and RDU markets. We further propose an algorithm to numerically obtain competitive equilibria based on discretization, which works for both the DU-comonotone market and the RDU-comonotone market. Although the comonotone and complete markets are closely related, many of our findings are intriguing and in sharp contrast to results in the literature on complete markets in terms of existence, uniqueness, and closed-form solutions of the equilibria, and comonotonicity of the pricing kernel.
\end{abstract}

Fangda Liu is supported by the NNSF of China (No. 11601540) and the Natural Sciences and Engineering Research Council of Canada (RGPIN-2020-04717, DGECR-2020-00340). Ruodu Wang is supported by the Natural Sciences and Engineering Research Council of Canada (RGPIN-2018-03823,

RGPAS-2018-522590).

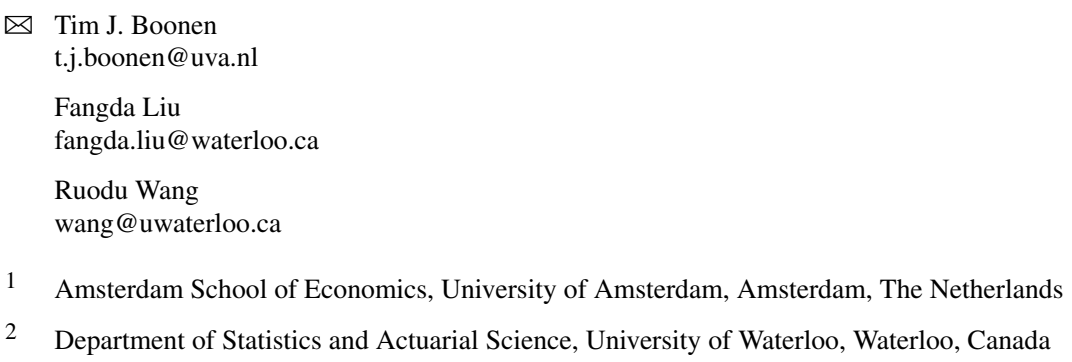


Keywords Competitive equilibria · Comonotone market · Dual utilities · Rank-dependent utilities · Pricing kernel

JEL Classification D52 - D86 · G10 · G22

\section{Introduction}

\subsection{Background}

This paper studies risk sharing games in a special type of one-period exchange markets, called the comonotone markets, and compare them with those in the classic complete markets. A comonotone market is one in which only comonotonic risk allocations (defined in Sect. 2) are allowed. Equivalently, market participants only trade risk allocations in the market that are comonotonic with the market total risk. This may be interpreted as, for instance, the market total risk is the systematic risk that is present, and participants are not allowed to bet against it.

Comonotone markets are closely related to the following economic setups. First, in the context of insurance risk allocations, comonotonic contracts are desirable a priori to the risk-exchange (e.g. Huberman et al. 1983). In particular, for comonotonic contracts, no insurer or insured would benefit from an incremental loss, and no participant has an incentive to underreport their losses. As a policyholder would have no incentive to increase the underlying loss in this case, comonotonicity is often referred to as the no-sabotage condition (e.g. Carlier and Dana 2003). In optimal reinsurance contract theory, comonotonicity of risk allocations implies that individual and aggregate indemnity contracts are not allowed to increase more than the underlying insured risk (e.g. Cheung et al. 2014 and Xu et al. 2019). Therefore, studying competitive equilibria in comonotone markets helps to understand the insurance/reinsurance market. Alternatively, suppose there is an insurance market, where all individuals face risk driven by a single factor. For instance, the individuals occur losses in case of a flood, an earthquake, but one can also think about a single default event of a major creditor. The individuals in the market can buy insurance against this common factor, but cannot bet against it. This gives rise to comonotone markets. Second, in collective risk sharing, the no-sabotage condition is an intuitive condition to impose as a property of risk allocations. It means that all agents in a collective agree to take positions that are non-decreasing functions of the total risk. This monotonicity property also plays a central role in cost sharing, where the cost of a good needs to be allocated among agents that each have a demand for this good. For instance, Moulin (1987) and Moulin and Shenker (1992) both study monotonicity properties in a deterministic setting. Moulin (1987) requires that the allocated costs weakly increase for all agents if the cost of the good increases. Moulin and Shenker (1992) impose that if an agent requires a higher amount of the good, then the cost allocation should weakly increase as well. Third, in a complete financial market with homogeneous beliefs, equilibrium allocations are generally comonotonic, and each position in an equilibrium allocation is counter-comonotonic with respect to the pricing kernel (see a detailed explanation in Sect. 2). A natural question is what happens if we constrain up-front the feasi- 
ble set of allocations to be comonotonic, and whether and how this would affect the equilibrium price and allocation. As such, the study of comonotone markets helps to enhance the understanding of the mechanism in complete markets, and this model is able to accommodate some intriguing phenomena such as the pricing kernel puzzle. Regardless of the interpretation one takes, our market setup is generic, and we shall simply refer to a market participant, be it an investor, a firm, an insured, an insurer or a reinsurer, as an agent.

In this paper, the preferences of agents are modelled by dual utilities (DU, Yaari 1987) or rank-dependent utilities (RDU, Quiggin 1982, 1993). These preference models received considerable attention as an alternative (and generalization) to expected utility, as RDU are one of the most popular decision models in behavioral economics. DU, as a special case of RDU, are related to the theory of risk measures (Artzner et al. 1999), which has gained great popularity after the introduction of the Basel III regulations for banks (e.g. Embrechts et al. 2014). The key feature of RDU is the non-linearity in the probabilities of the risk evaluation. We focus on RDU preferences because, even in a complete market, finding competitive equilibria is very challenging; existence may not be guaranteed, and explicit forms are generally unavailable. This is in sharp contrast to the case of expected utilities in a complete market, where existence and explicit forms are available.

Recent advances on RDU equilibria in complete markets are summarized in Xia and Zhou (2016) and Jin et al. (2019). Our paper is essentially different from Xia and Zhou (2016) and Jin et al. (2019), noting that our market is comonotone to begin with. In the latter two papers, non-trivial technical assumptions are imposed to guarantee the existence of an RDU equilibrium, and this also ensures counter-comonotonicity of the pricing kernel with the market risk. In our paper, we show that the pricing kernel is not necessarily counter-comonotonic with the market risk, and we require only a very weak condition on the structure of the preferences to guarantee existence. See Sect. 4 for more detailed discussions on the differences between our results and those in the classic complete market model. We shall see that in the comonotone market we consider, the problem becomes much more mathematically tractable, because comonotonicity yields technical convenience allowing one to translate between RDU and an expectedutility-based model with heterogeneous beliefs. Nevertheless, in order for explicit calculation of competitive equilibria, some non-trivial technical conditions need to be imposed.

\subsection{Contribution of the paper}

The main part of in this paper is dedicated to finding competitive equilibria in a comonotone market, discussing their economic properties, and comparing them with the case of a complete market. In Sect. 2, we formally introduce the comonotone market, collect its properties and its relation to the corresponding complete market, and review DU and RDU preference models.

Section 3 contains our main results on markets with DU agents. In complete markets, competitive equilibria do not necessarily exist unless the probability distortions are strictly convex. In comonotone markets, we show the existence of competitive 
equilibria, and we provide complete closed-form solutions. Generally, the equilibrium price is not necessarily unique. If probability distortions are non-convex, the equilibrium pricing kernel does not need to be counter-comonotonic with the market risk. If the probability distortions are different almost everywhere, then the equilibrium risk allocations are unique up to constants. Moreover, we show that the two classic fundamental theorems of welfare economics hold in comonotone markets with DU agents.

Section 4 addresses markets with RDU agents. In complete markets, once again competitive equilibria do not need to exist unless the probability distortions are strictly convex. Strict convexity of distortion functions is consistent with strong risk aversion (Chew et al. 1987). In contrast, we establish the existence of competitive equilibria in comonotone markets. This is a result of the fact that our preferences are strictly convex on the set of comonotonic allocations, and thus the agents are strong risk averse. Moreover, we establish the two fundamental theorems of welfare economics in comonotone markets. Although closed-form solutions of competitive equilibria are not universally available, we do obtain them if the distortions are considered to be "close" to each other. In particular, we obtain an analytical condition for RDU agents with exponential utilities.

To compute competitive equilibria in a comonotone market with general DU and RDU agents, especially in the cases where explicit solutions are not available, we design an efficient algorithm in Sect. 5, and its numerical performance is reported in Sect. 6 with various examples.

In general, comonotone markets allows for a flexible framework to accommodate interesting phenomena. In complete markets, the equilibrium pricing kernel is necessarily counter-comonotonic with the market risk. This is a reason that competitive equilibria do not necessarily exist for rank-dependent utilities (Jin et al. 2019) or the more general cumulative prospect theory preferences (De Giorgi et al. 2010). In comonotone markets, the equilibrium pricing kernel does not need to be countercomonotonic with the market risk, and existence of the equilibrium is guaranteed under mild conditions.

\subsection{Related literature}

There is a relatively large literature that analyzes competitive equilibria in risk sharing, based on the seminal work of Arrow and Debreu (1954). Pareto-optimal and competitive risk sharing in complete markets with (convex) risk measures (which include convex dual utilities) is studied by Heath and $\mathrm{Ku}$ (2004), Barrieu and El Karoui (2005), Jouini et al. (2008), and Dana and Le Van (2010); see Ludkovski and Young (2009) and Boonen $(2015,2017)$ for a more focused treatment on dual utilities. The non-convex case is studied recently for quantile-based risk measures (special cases of dual utilities) by Embrechts et al. (2018) and Embrechts et al. (2020).

Risk sharing with rank-dependent utilities is studied by, amongst others, Chateauneuf et al. (2000) and Tsanakas and Christofides (2006), who also assume convex probability distortions. In this paper, we consider general probability distortions (not necessarily concave or convex), that include inverse-S shaped distortions proposed 
by Tversky and Kahneman (1992). Competitive equilibria in complete markets for not necessarily convex distortions are studied recently by Xia and Zhou (2016) and Jin et al. (2019), who establish some sufficient conditions for the existence of equilibria.

A key concept we introduce and study in this paper is the comonotone market. A summary of comonotonicity and its related properties can be found in Denuit et al. (2005). The profound connection between optimality in risk sharing and comonotonicity is studied in Landsberger and Meilijson (1994) and Ludkovski and Rüschendorf (2008); see Rüschendorf (2013) and the references therein. Because of the imposed comonotonicity, a comonotone market with RDU agents is closely related to a complete market with expected utility agents and heterogeneous beliefs. Risk sharing in complete markets with expected utilities gained interest already by Arrow and Debreu (1954), and have been generalized by many authors thereafter (e.g. Borch 1962; Wilson 1968; Dana 1993). An extension to dynamic risk sharing under a notion of ambiguity is proposed by Dana and Riedel (2013). A more recent account is given by Anthropelos and Kardaras (2017), where agents can strategically choose their subjective beliefs regarding the underlying risk in the market. A more common notion of market incompleteness arises by the spanning of the payoff space by a limited number of securities. For instance, Araujo et al. (2012) and Beissner and Riedel (2019) study incomplete markets with sublinear price functionals, that are a result of transaction costs. Also, market incompleteness by constraints on feasible risk sharing contracts has particularly gained popularity in the financial literature on short-selling constraints, which is a weaker constraint than the constraints in comonotone markets (Jarrow 1980; Nielsen 1989; Heaton and Lucas 1996). Our use of comonotone markets is not driven by constraints on securities, but arise by directly constraining the payoff space. As far as we are aware of, this paper is the first one to formulate market incompleteness by means of the comonotone market and to study risk sharing in this market. In comonotone markets, we assume that our pricing function is comonotonic additive, which is introduced by Schmeidler (1986).

Another related stream of research is in the literature of optimal insurance/reinsurance design, where comonotonicity of risk allocation is assumed as a natural requirement of no moral hazard. Our technical approaches share some similarity with recent developments in this field; for the latter, the reader is referred to Bernard et al. (2015), Cai et al. (2016), Xu et al. (2019) and the references therein.

\section{Notation and preliminaries}

\subsection{Dual utilities and rank-dependent utilities}

We work with a probability space $(\Omega, \mathcal{B}, \mathbb{P})$, and denote the set of bounded random variables $^{1}$ on it by $\mathcal{X}=L^{\infty}(\Omega, \mathcal{B}, \mathbb{P})$. We consider a one-period exchange market with a finite set of agents, given by $N=\{1, \ldots, n\}$. Each agent in the market is endowed with an endowment $\xi_{i} \in \mathcal{X}, i \in N$, that we interpret as a (random) future

\footnotetext{
1 The reason of working with bounded random variables in this paper is simply to avoid possible infinity in optimizations. Most results can be naturally generalized to unbounded random variables, assuming that the preference functionals we encounter take finite values on these random variables.
} 
wealth at a given future reference period. The total (future) wealth in the market is then given by $X=\sum_{i=1}^{n} \xi_{i}$, and denote by $R(X)$ the range of $X$, which is assumed to be a closed real interval. ${ }^{2}$ We assume that $\mathcal{B}$ is the $\sigma$-algebra generated by $X$. Throughout, $F_{X}(\cdot)=\mathbb{P}(X \leq \cdot)$ represents the cumulative distribution function of a random variable $X$ and $S_{X}=1-F_{X}$ represents its survival function.

In this paper, we focus on rank-dependent utilities (RDU) (Quiggin 1982, 1993) as class of risk preferences, which includes dual utilities (DU) (Yaari 1987) as special cases. Define the set of distortion functions (also called probability perception functions $)^{3}$

$\mathcal{G}=\{g:[0,1] \rightarrow[0,1] \mid g$ is continuous and increasing, $g(0)=0$ and $g(1)=1\}$.

A dual utility functional $D_{g}$ with distortion function $g \in \mathcal{G}$ is defined as a Choquet integral (see e.g. Denneberg 1994 and Wang et al. 1997), namely,

$$
D_{g}(Y)=\int Y \mathrm{~d}(g \circ \mathbb{P}):=\int_{-\infty}^{0}\left(g\left(S_{Y}(z)\right)-1\right) \mathrm{d} z+\int_{0}^{\infty} g\left(S_{Y}(z)\right) \mathrm{d} z, \quad Y \in \mathcal{X} .
$$

For an increasing function $u: \mathbb{R} \rightarrow[-\infty, \infty)$ and a distortion function $g \in \mathcal{G}$, a rank-dependent utility functional $R_{u, g}$ is given by

$$
R_{u, g}(Y)=D_{g}(u(Y))=\int u(Y) \mathrm{d}(g \circ \mathbb{P}), \quad Y \in \mathcal{X} .
$$

Throughout, we shall use the abbreviations DU for dual utility functionals, and RDU for rank-dependent utility functionals. Note that DU does not take the value $-\infty$ on $\mathcal{X}$ whereas RDU may take the value $-\infty$ on a subset of $\mathcal{X}$ depending on the choice of $u$, such as power utility functions.

RDU is a special case of Choquet expected utility, in which the agent's non-additive measure (also called a capacity) $v$ is a distortion of a probability measure $(v=g \circ \mathbb{P})$. In this case, convexity of the probability weighting function $g$ yields convexity of the capacity $v$. In Choquet expected utility, a convex capacity reflects ambiguity aversion, and a concave capacity reflects an ambiguity-seeking behavior. It is well known that $R_{u, g}$ is consistent with strong risk aversion. ${ }^{4}$ if and only if $u$ is concave and $g$ is convex. Due to the popularity of and the empirical evidence for non-convex distortion functions in modern decision theory (e.g. Tversky and Kahneman 1992 and Quiggin 1993), we are particularly interested in the cases where $g_{1}, \ldots, g_{n}$ are not necessarily convex.

\footnotetext{
${ }^{2}$ For instance, if $X$ is a uniform random variable on $(0,1)$, then $R(X)=[0,1]$.

${ }^{3}$ For the ease of presentation, we only consider continuous distortion functions, although many results can be applied to the case of discontinuous distortion functions.

${ }^{4}$ This result was shown by Chew et al. (1987) For risk aversion in more advanced decision models, see Schmidt and Zank (2008) and Müller et al. (2017).
} 


\subsection{Comonotone market and competitive equilibria}

A pair of random variables $(Y, Z) \in \mathcal{X}^{2}$ is called comonotonic, or equivalently, the pair $(Y,-Z)$ is called counter-comonotonic, if $Y=f(Y+Z)$ and $Z=g(Y+Z)$ for some increasing functions ${ }^{5} f$ and $g$. We say that a random vector $\left(X_{1}, \ldots, X_{n}\right)$ is comonotonic if each pair of $X_{1}, \ldots, X_{n}$ is comonotonic. Equivalently, there exist increasing functions $f_{1}, \ldots, f_{n}$ such that $X_{i}=f_{i}\left(X_{1}+\cdots+X_{n}\right), i=1, \ldots, n$.

Denote by $\mathcal{P}$ the set of all probability measures on $(\Omega, \mathcal{B})$ which are absolutely continuous with respect to $\mathbb{P}$. The current price of a random wealth $Y$ in the market is given by $\mathbb{E}^{\mathbb{Q}}[Y]$ for some $\mathbb{Q} \in \mathcal{P}$, which we shall refer to as a pricing measure.

We consider competitive equilibria in two market settings. The main focus is a special type of incomplete market, the comonotone market, in which allocations are confined to the set $C(X)$ of comonotonic allocations, namely,

$$
C(X)=\{Y \in \mathcal{X}:(Y, X-Y) \text { is comonotonic }\}
$$

In this market, the set of admissible allocations ${ }^{6}$ is denoted by

$$
\mathbb{A}_{n}^{c}(X)=\left\{\left(X_{1}, \ldots, X_{n}\right) \in(C(X))^{n}: \sum_{i=1}^{n} X_{i}=X\right\}
$$

For comparison with the comonotone market, we also consider a complete market, where the set of admissible allocations is denoted by

$$
\mathbb{A}_{n}(X)=\left\{\left(X_{1}, \ldots, X_{n}\right) \in \mathcal{X}^{n}: \sum_{i=1}^{n} X_{i}=X\right\}
$$

The preference of agent $i \in N$ is modelled via an objective functional $V_{i}$, which is a dual utility or rank-dependent utility functional. In general, competitive equilibrium is a pricing measure and a risk redistribution, such that given the price formula, each agent $i \in N$ individually maximizes $V_{i}\left(X_{i}^{*}\right)$ such that $X_{i}^{*}$ is in the budget set, and the pricing measure $\mathbb{Q}$ induces market-clearing by equating aggregate supply and demand, i.e., $\sum_{i=1}^{n} X_{i}^{*}=X$. The formal definition of competitive equilibria in the two markets is given below. ${ }^{7}$

\footnotetext{
5 In this paper, terms "increasing" and "decreasing" are in the non-strict sense.

6 In fact, any tuple of random variables in $C(X)$ is comonotonic; see Denneberg (1994). According to Denneberg's Lemma (Denneberg 1994), for an allocation $\left(X_{1}, \ldots, X_{n}\right) \in \mathbb{A}_{n}^{c}(X)$ and each $i \in N$, it is possible to write $X_{i}=f_{i}(X)$ for some $f_{i} \in \mathcal{F}$. As a consequence, $\left(X_{1}, \ldots, X_{n}\right)$ is comonotonic. These facts are used repeatedly in the analysis of this paper.

7 In a market of finitely many linear assets, the existence of a pricing measure follows from the no-arbitrage assumption by the first Fundamental Theorem of Asset Pricing in the form of Theorem 1.7 of Föllmer and Schied (2016). In the more general markets such as the ones we consider in Definition 1, a small extra assumption of continuity is required, such as semi-continuity of the pricing functional with respect to the weak* topology (see e.g., Theorem 12 of Delbaen 2012). Following the standard practice in the literature, we directly assume that the pricing in the market is induced by probability measures.
} 
Definition 1 (Competitive equilibria) Fix objective functionals $V_{1}, \ldots, V_{n}$, total risk $X \in \mathcal{X}$ and initial endowments $\xi_{1}, \ldots, \xi_{n} \in \mathcal{X}$, and define the budget set $B\left(\mathbb{Q}, \xi_{i}\right)=$ $\left\{Y \in \mathcal{X}: \mathbb{E}^{\mathbb{Q}}[Y] \leq \mathbb{E}^{\mathbb{Q}}\left[\xi_{i}\right]\right\}$.

(i) A constrained competitive equilibrium (CCE) is a pair of comonotonic allocation and pricing measure $\left(\left(X_{1}, \ldots, X_{n}\right), \mathbb{Q}\right) \in \mathbb{A}_{n}^{c}(X) \times \mathcal{P}$ such that for $i \in N$,

$$
X_{i} \in B\left(\mathbb{Q}, \xi_{i}\right) \text { and } V_{i}\left(X_{i}\right)=\max \left\{V_{i}\left(Y_{i}\right): Y_{i} \in C(X) \cap B\left(\mathbb{Q}, \xi_{i}\right)\right\}
$$

(ii) An unconstrained competitive equilibrium (UCE) is a pair of allocation and pricing measure $\left(\left(X_{1}, \ldots, X_{n}\right), \mathbb{Q}\right) \in \mathbb{A}_{n}(X) \times \mathcal{P}$ such that for $i \in N$,

$$
X_{i} \in B\left(\mathbb{Q}, \xi_{i}\right) \text { and } V_{i}\left(X_{i}\right)=\max \left\{V_{i}\left(Y_{i}\right): Y_{i} \in B\left(\mathbb{Q}, \xi_{i}\right)\right\}
$$

In a competitive equilibrium, $\left(X_{1}, \ldots, X_{n}\right)$ is an equilibrium allocation, and $\mathbb{Q}$ is an equilibrium price. ${ }^{8}$

Clearly, if the allocation in a UCE is comonotonic, then the UCE is a CCE for the same set of objective functionals and initial endowments.

Competitive equilibria rely on the assumption that there is a competitive environment, where individual transactions have no influence on the prices. UCE in the complete market for rank-dependent utilities are studied recently in Xia and Zhou (2016) and Jin et al. (2019), and an analytical solution is very difficult to obtain in general for heterogeneous distortion functions. In this paper, our main focus is put on the comonotone market, and we shall investigate the difference between equilibria in these two markets, namely CCE and UCE.

Suppose that $\left(\left(X_{1}^{*}, \ldots, X_{n}^{*}\right), \mathbb{Q}\right)$ is a $\mathrm{UCE}$, and let $\eta=\mathrm{d} \mathbb{Q} / \mathrm{d} \mathbb{P}$, namely the pricing kernel. ${ }^{9}$ As one of the classic results in equilibrium asset pricing theory in complete markets (e.g. Föllmer and Schied 2016 and Jin et al. 2019), assuming that the objectives are law-determined and strictly monotone and $\eta$ is continuously distributed, the following statements hold.

(i) $\left(X_{i}^{*}, \eta\right)$ is counter-comonotonic (Dybvig 1988).

(ii) $(X, \eta)$ is counter-comonotonic (a result of (i)).

(iii) $\left(X_{1}^{*}, \ldots, X_{n}^{*}\right) \in \mathbb{A}_{n}^{c}(X)$ (a result of (i)).

The above fact (ii) is commonly referred to as the law of demand and supply, meaning that in an equilibrium, the higher supply (larger values of $X$ ) is associated with a lower price (smaller values of $\eta$ ); see e.g. Chabi-Yo (2012) and Hens and Reichlin (2013). Therefore, from (iii), under mild conditions, a UCE is always a CCE. However, from the detailed analysis in this paper, we shall see that a CCE is not necessarily a UCE. Moreover, for a $\operatorname{CCE}\left(\left(X_{1}^{*}, \ldots, X_{n}^{*}\right), \mathbb{Q}\right)$, the above (i) and (ii) are not necessarily true, thus in sharp contrast to the case of complete market.

A concept closely related to competitive equilibria is the classic Pareto optimality, also known as Pareto efficiency.

\footnotetext{
${ }^{8}$ It may happen that $V_{i}\left(X_{i}\right)=-\infty$ in both (i) and (ii). This may be, for instance, the case where an agent with a logarithmic utility function has a negative endowment. We shall mainly focus on the cases where $V_{i}\left(X_{i}\right)>-\infty$.

${ }^{9}$ Since $\mathbb{E}^{\mathbb{Q}}[1]=1$, we know that the pricing kernel $d \mathbb{Q} / d \mathbb{P}$ is in $L^{1}(\Omega, \mathcal{B}, \mathbb{P})$.
} 
Definition 2 (Pareto-optimal allocations) Fix objective functionals $V_{1}, \ldots, V_{n}$, total risk $X \in \mathcal{X}$ and initial endowments $\xi_{1}, \ldots, \xi_{n} \in \mathcal{X}$.

(i) In the comonotone market, an allocation $\left(X_{1}, \ldots, X_{n}\right) \in \mathbb{A}_{n}^{c}(X)$ is Paretooptimal if for any allocation $\left(Y_{1}, \ldots, Y_{n}\right) \in \mathbb{A}_{n}^{c}(X), V_{i}\left(Y_{i}\right) \geq V_{i}\left(X_{i}\right)$ for $i \in N$ implies $V_{i}\left(Y_{i}\right)=V_{i}\left(X_{i}\right)$ for $i \in N$.

(ii) In the complete market, an allocation $\left(X_{1}, \ldots, X_{n}\right) \in \mathbb{A}_{n}(X)$ is Pareto-optimal if for any allocation $\left(Y_{1}, \ldots, Y_{n}\right) \in \mathbb{A}_{n}(X), V_{i}\left(Y_{i}\right) \geq V_{i}\left(X_{i}\right)$ for $i \in N$ implies $V_{i}\left(Y_{i}\right)=V_{i}\left(X_{i}\right)$ for $i \in N$.

The main part of this paper is dedicated to derive CCE, compare it with properties of UCE, and establish its relation to Pareto-optimal allocations through the fundamental theorems of welfare economics.

Note that the classic von Neumann-Morgenstern expected utility functional (EU) is a special case of RDU by taking $g$ as the identity function on $[0,1]$. Moreover, a DU is a special case of an RDU by taking $u$ as the identity function. In what follows we treat the corresponding CCE problems with DU and RDU separately in Sects. 3 and 4 for two reasons. First, we can analytically solve CCE for DU, but not for RDU, and second, in this paper we only consider RDU in which $u$ is strictly concave, a common setup in the study of RDU (Quiggin 1993), which rules out DU as a special case; see Sect. 4.

\subsection{Individual optimization}

In a comonotone market, the individual optimization problem is to find $X_{i}^{*}$ which solves

$$
\begin{aligned}
& \max _{X_{i} \in C(X) \cap B\left(\mathbb{Q}, \xi_{i}\right)} V_{i}\left(X_{i}\right) \text {, or, equivalently, } \\
& \max _{X_{i} \in C(X)} V_{i}\left(X_{i}\right) \text { s.t. } \mathbb{E}^{\mathbb{Q}}\left[X_{i}\right] \leq \mathbb{E}^{\mathbb{Q}}\left[\xi_{i}\right] .
\end{aligned}
$$

If $V_{i}, i \in N$, are DU or RDU, we first present a simple fact on the individual optimization problem (2), which will serve as a building block to solving the CCE in Sects. 3 and 4 and as the basis of the numerical algorithm in Sect. 5.

Lemma 1 Let $V_{i}=R_{u_{i}, g_{i}}, i \in N$, where $u_{i}, i \in N$, are strictly increasing functions. For a fixed $\mathbb{Q}$ and $X_{i} \in C(X)$, let $Y_{i}=X_{i}-\mathbb{E}^{\mathbb{Q}}\left[\xi_{i}\right]+\mathbb{E}^{\mathbb{Q}}\left[Y_{i}\right]$. Then, $X_{i}$ maximizes problem (2) if and only if $Y_{i}$ satisfies

$$
Y_{i} \in \underset{Y \in C(X)}{\arg \max }\left\{V_{i}\left(Y-\mathbb{E}^{\mathbb{Q}}[Y]+\mathbb{E}^{\mathbb{Q}}\left[\xi_{i}\right]\right)\right\}
$$

The proof of the lemma follows directly from the fact that the budget constraint $\mathbb{E}^{\mathbb{Q}}[Y] \leq \mathbb{E}^{\mathbb{Q}}\left[\xi_{i}\right]$ in $B\left(\mathbb{Q}, \xi_{i}\right)$ is binding, because of the strict monotonicity of the objective $V_{i}$. 
In the special case of $V_{i}=D_{g_{i}}$ for an individual agent (that is, $u_{i}$ is the identity function), for a fixed $\mathbb{Q},(3)$ boils down to

$$
Y_{i} \in \underset{Y \in C(X)}{\arg \max }\left\{V_{i}(Y)-\mathbb{E}^{\mathbb{Q}}[Y]\right\} .
$$

\section{Competitive equilibria with dual utilities}

In this section, we study the case of equilibria for dual utilities. Throughout this section, we impose the following assumption.

Assumption 1 Let $V_{i}=D_{g_{i}}$ for $i \in N$, where $g_{1}, \ldots, g_{n} \in \mathcal{G}, \xi_{1}, \ldots, \xi_{n} \in \mathcal{X}$, and $X=\sum_{i=1}^{n} \xi_{i}$.

For the convenience of presentation, we shall refer a market with the above objectives and endowments as a DU-comonotone market or a DU-complete market.

\subsection{Equilibrium allocations and prices}

Let

$$
\mathcal{F}=\left\{f: \mathbb{R} \rightarrow \mathbb{R} \mid f \text { is absolutely continuous, } 0 \leq f^{\prime}(z) \leq 1 \text { for } z \in \mathbb{R}\right\}
$$

where for $f \in \mathcal{F}$, we use $f^{\prime}$ to represent a function that is almost everywhere (a.e.) equal to the derivative of $f .{ }^{10}$

In the case of a DU-complete market where the distortion functions are not convex, a UCE does not necessarily exist; see Embrechts et al. (2018) in the case of quantiles. Let $g_{N, 1}(t)=\max \left\{g_{1}(t), \ldots, g_{n}(t)\right\}$ for $t \in[0,1]$. If the distortion functions are all convex, then a UCE in a complete market $\left(\left(X_{1}^{*}, \ldots, X_{n}^{*}\right), \mathbb{Q}\right)$ exists, and is given by $\mathbb{Q}(X>z)=g_{N, 1}\left(S_{X}(z)\right)$ for all $z \in R(X)$ and $X_{i}^{*}=f_{i}(X)$ such that $f_{i} \in \mathcal{F}$, and for all $z \in R(X)$ :

$$
f_{i}^{\prime}(z)=0 \text {, if } g_{i}\left(S_{X}(z)\right)<\mathbb{Q}(X>z) \text { and } \sum_{i=1}^{n} f_{i}^{\prime}(z)=1 \text {. }
$$

We refer to Boonen (2015) for the case of a finite state space.

We proceed with studying the DU-comonotone market. For a fixed $\mathbb{Q}$, we first give an explicit solution to the individual optimization problem (2) based on Lemma 1.

Proposition 1 Suppose that Assumption 1 holds. For a fixed $\mathbb{Q}$ and $f_{i} \in \mathcal{F}$, the random variable $Y_{i}=f_{i}(X)$ satisfies (4) if and only if for all $z \in R(X)$,

$$
f_{i}^{\prime}(z)=1 \text {, if } g_{i}\left(S_{X}(z)\right)>\mathbb{Q}(X>z) \text {, and } f_{i}^{\prime}(z)=0 \text {, if } g_{i}\left(S_{X}(z)\right)<\mathbb{Q}(X>z)
$$

\footnotetext{
10 When speaking of derivatives of functions in $\mathcal{F}$, we do not distinguish two functions that are a.e. equal.
} 
Combining Proposition 1 and Lemma 1, we see that there always exists a maximizer to the individual optimization problem (2) given by $X_{i}=Y_{i}-\mathbb{E}^{\mathbb{Q}}\left[Y_{i}\right]+\mathbb{E}^{\mathbb{Q}}\left[\xi_{i}\right]$.

We continue by characterizing the CCE explicitly. In the following, $g_{N, 2}(t)$ is the second-largest element in $\left\{g_{1}(t), \ldots, g_{n}(t)\right\}$ for $t \in[0,1]$.

Theorem 1 In the comonotone market under Assumption 1 , the pair $\left(\left(X_{1}^{*}, \ldots, X_{n}^{*}\right), \mathbb{Q}\right)$ is a CCE if and only if the following hold

(i) $g_{N, 2}\left(S_{X}(z)\right) \leq \mathbb{Q}(X>z) \leq g_{N, 1}\left(S_{X}(z)\right)$ for $z \in R(X)$;

(ii) for $i \in N, X_{i}^{*}=f_{i}(X)-\mathbb{E}^{\overline{\mathbb{Q}}}\left[f_{i}(X)\right]+\mathbb{E}^{\mathbb{Q}}\left[\xi_{i}\right]$ almost surely where $f_{i}$ satisfies (5) with $\sum_{i=1}^{n} f_{i}(X)=X$.

If there exists an $i \in N$ such that $g_{i}(t)=g_{N, 1}(t)$ for all $t \in[0,1]$, then we interpret this agent as the most risk-loving agent since $D_{g_{i}}(Y) \geq D_{g_{N, 2}}(Y) \geq D_{g_{j}}(Y)$ for every risk $Y \in \mathcal{X}$ and every other $j \in N$. Therefore, there exists an equilibrium such that agent $i$ bears all the aggregate risk in equilibrium and the other agents only face deterministic risk allocations (e.g., full insurance in an insurance setting). If there does not exist a most risk-loving agent, then locally a most-risk loving agent bears the local aggregate risk. More precisely, the marginal risk of $X$ at $z \in R(X)$ is allocated to the $\operatorname{agent}(\mathrm{s})$ in $\mathcal{I}(z)=\left\{i \in N: g_{i}\left(S_{X}(z)\right)=g_{N, 1}\left(S_{X}(z)\right)\right\}$ and $\sum_{i \in \mathcal{I}(z)} f_{i}^{\prime}(z)=1$.

From Theorem 1, we get directly the following corollary for the case of identical dual utilities among agents.

Corollary 1 In the comonotone market under Assumption 1 , if $g_{i}=g$ for all $i \in N$, then the pair $\left(\left(X_{1}^{*}, \ldots, X_{n}^{*}\right), \mathbb{Q}\right)$ is a CCE if and only if the following hold

(i) $\mathbb{Q}(X>z)=g\left(S_{X}(z)\right)$ for $z \in R(X)$;

(ii) for $i \in N, X_{i}^{*}=f_{i}(X)-\mathbb{E}^{\mathbb{Q}}\left[f_{i}(X)\right]+\mathbb{E}^{\mathbb{Q}}\left[\xi_{i}\right]$ almost surely where $\sum_{j=1}^{n} f_{j}(X)=X$ and $f_{i} \in \mathcal{F}$.

According to Corollary 1, if all agents use the same DU, then the equilibrium price turns out to be unique. On the contrary, equilibrium allocations are not unique. In fact, each comonotonic allocation satisfying the individual budget constraints is an equilibrium allocation. In the theorem below we analyze existence and uniqueness of the CCE in more details.

Theorem 2 In the comonotone market under Assumption 1, the following hold.

(i) A CCE always exists.

(ii) If $g_{N, 1}(t)>g_{N, 2}(t)$ for almost everywhere $t \in[0,1]$, then the equilibrium (CCE) allocation is unique up to constant shifts, and the equilibrium price is not unique.

(iii) If $g_{N, 1}(t)=g_{N, 2}(t)$ for almost everywhere $t \in[0,1]$, then the equilibrium (CCE) price is unique, and the equilibrium allocation is not unique.

In (i) of the theorem, we obtain existence of a CCE in a comonotone market. We wish to point out that existence of a UCE may not hold in expected utility complete markets with an unbounded commodity space (e.g. Cheng 1991 and Dana and Le Van 2000). Assumptions in such markets to obtain existence of equilibria often include that individual agents are risk-averse or the individually rational utility set is compact. In 
our DU-comonotone market, such assumptions are not needed. In (ii) of the theorem, by saying that the equilibrium allocation is unique up to constant shifts, we mean that, for any two CCEs $\left(\left(X_{1}, \ldots, X_{n}\right), \mathbb{Q}\right)$ and $\left(\left(Y_{1}, \ldots, Y_{n}\right), \tilde{\mathbb{Q}}\right), X_{i}-Y_{i}, i \in N$ are deterministic. Here, the two pricing measures $\mathbb{Q}$ and $\tilde{\mathbb{Q}}$ may not be the same. In particular, $X_{i}-Y_{i}=\mathbb{E}^{\mathbb{Q}}\left[\xi_{i}-Y_{i}\right]=\mathbb{E}^{\tilde{\mathbb{Q}}}\left[X_{i}-\xi_{i}\right]$.

By constraining the market by allowing only for comonotonic allocations, the equilibrium price is typically not unique, and the difference with a complete market can be explained intuitively as follows. In a complete market, suppose we have $\mathbb{Q}(X>z)<g_{1}\left(S_{X}(z)\right)$ for some $z$ and $X_{1} \in C(X)$. Then, we let Agent 1 buy the security $\delta\left(\mathbb{I}_{\{X>z\}}-\mathbb{Q}(X>z)\right)$ for $\delta>0$, where $\mathbb{I}_{A}$ is the indicator function of an event $A$. Note that the resulting new position $X_{1}^{*}=X_{1}+\delta\left(\mathbb{I}_{\{X>z\}}-\mathbb{Q}(X>z)\right)$ has the same price as $X_{1}$, and it increases the utility of Agent 1 by $\delta\left(g_{1}\left(S_{X}(z)\right)-\mathbb{Q}(X>z)\right)>0$. Infinite profit for Agent 1 follows from letting $\delta \rightarrow \infty$, making an equilibrium allocation impossible. On the other hand, in a comonotone market, the feasible choice of $\delta$ is constrained. Hence, this strategy with asymptotic profits is not feasible anymore. This fact leads to the sharp qualitative contrast between the two markets.

\subsection{Pareto optimality and fundamental theorems of welfare economics}

In this section we discuss the relation between equilibria and Pareto optimality, and establish fundamental theorems of welfare economics for the DU-comonotone market. First, we state a simple fact that for DU agents, Pareto optimality is equivalent to optimality with respect to the sum. This result is well known (see e.g. Proposition 1 of Embrechts et al. 2018) in case of the complete market, and the same argument therein applies to the comonotone market.

Proposition 2 In the comonotone market under Assumption 1 , an allocation $\left(X_{1}, \ldots\right.$, $\left.X_{n}\right) \in \mathbb{A}_{n}^{c}(X)$ is Pareto-optimal if and only if it is sum-optimal, that is,

$$
\sum_{i=1}^{n} V_{i}\left(X_{i}\right)=\max \left\{\sum_{i=1}^{n} V_{i}\left(Y_{i}\right):\left(Y_{1}, \ldots, Y_{n}\right) \in \mathbb{A}_{n}^{c}(X)\right\} .
$$

The value of the right-hand side of (6) can be easily calculated. Indeed, by Proposition 5 of Embrechts et al. (2018), we have, for any $S \subseteq N$ and $Y \in C(X)$,

$$
\max \left\{\sum_{i \in S} V_{i}\left(Y_{i}\right): Y_{i} \in C(X), \sum_{i \in S} Y_{i}=Y\right\}=D_{g_{S, 1}}(Y),
$$

where $D_{g_{S, 1}}$ is the dual utility with distortion function $g_{S, 1}(s)=\max \left\{g_{i}(s): i \in S\right\}$ for $s \in[0,1]$. Hence, from Theorem 1 that for every CCE $\left(\left(X_{1}^{*}, \ldots, X_{n}^{*}\right), \mathbb{Q}\right)$, we get

$$
\sum_{i=1}^{n} V_{i}\left(X_{i}^{*}\right)=D_{g_{N, 1}}(X)
$$


and

$V_{i}\left(X_{i}^{*}\right) \geq \mathbb{E}^{\mathbb{Q}}\left[X_{i}^{*}\right] \geq D_{g_{N \backslash\{i\}, 1}}\left(X_{i}^{*}\right)=\max \left\{\sum_{j \neq i} V_{j}\left(Y_{j}\right): Y_{j} \in C\left(X_{i}^{*}\right), \sum_{j \neq i} Y_{j}=X_{i}^{*}\right\}$.

Note that $D_{g_{N \backslash\{i\}, 1}}\left(X_{i}^{*}\right)$ is the maximum price that all agents, except $i$, are jointly willing to offer to bear the risk $X_{i}^{*}$.

We conclude the section by establishing the fundamental theorems of welfare economics (FTWE) for the DU-comonotone market. The proof of FTWE relies on the explicit form of CCEs obtained in Theorem 1 and the relation to sum-optimality in Proposition 2.

Theorem 3 In the comonotone market under Assumption 1, the following hold.

(i) An equilibrium (CCE) allocation is necessarily Pareto-optimal.

(ii) A Pareto-optimal allocation is necessarily an equilibrium (CCE) allocation for some choice of endowments.

\section{Competitive equilibria with rank-dependent utilities}

We proceed to the market with agents using rank-dependent utilities, which is a much more complicated object to analyze. Throughout this section we make the following assumption.

Assumption 2 Let $V_{i}=R_{u_{i}, g_{i}}$ for $i \in N$, where $g_{1}, \ldots, g_{n} \in \mathcal{G}$ are continuously differentiable, $u_{1}, \ldots, u_{n}$ are strictly increasing and strictly concave utility functions, $\xi_{1}, \ldots, \xi_{n} \in \mathcal{X}$, and $X=\sum_{i=1}^{n} \xi_{i}$.

For $i \in \mathbb{N}$, write $d_{i}=\inf \left\{x \in \mathbb{R}: u_{i}(x)>-\infty\right\}$, that is, the infimum wealth value on which the utility of agent $i$ is finite, and assume that $u_{i}$ is continuously differentiable on $\left(d_{i}, \infty\right)$. We say an allocation $\left(X_{1}, \ldots, X_{n}\right)$ is proper if $V_{i}\left(X_{i}\right)>-\infty$ for all $i \in N$. Certainly, only proper allocations are economically interesting. For the convenience of presentation, we shall refer a market with the above objectives and endowments as an RDU-comonotone market or an RDU-complete market.

\subsection{General results}

We start with stating equilibrium results in the RDU-complete market. UCE do not need to exist when distortions are not convex (see Xia and Zhou 2016 and Jin et al. 2019). For example, in Xia and Zhou (2016), if the homogeneous distortion function $g_{i}=g$ is not concave, the assumption that the function $g^{\prime}(1-t) u^{\prime}\left(\operatorname{VaR}_{X}(t)\right)^{11}$ is strictly increasing in $t$ is required to guarantee the existence of equilibria. Xia and Zhou (2016) consequently show counter-comonotonicity between the pricing kernel

\footnotetext{
11 On top of individual agents, Xia and Zhou (2016) define a representative agent in the market, and $u$ is the utility of the representative agent.
} 
and the market total wealth. Moreover, Jin et al. (2019) show existence of UCE under a technical condition that implies the counter-comonotonicity between the pricing kernel and the market total wealth.

We proceed with studying the RDU-comonotone market. In Lemma 1, the individual optimization problem (2) is linked to looking for $Y_{i} \in C(X)$ satisfying (3). Our first result shows the existence of such $Y_{i}$.

Proposition 3 In the comonotone market under Assumption 2, there exists $Y_{i} \in C(X)$ satisfying (3). Thus, the individual maximal objectives in (2) are achieved.

We next establish the existence of a CCE, as well as the first and second FTWE in this market. The proof of the following results hinges on the observation that the preference $V_{i}=R_{u_{i}, g_{i}}$ is continuous and concave on the domain $C(X)$, and $C(X)$ is a convex set. Then, one builds on the existing results of existence and FTWE in discrete models, e.g. Theorem 1 of Werner (1987) and Propositions 16.D.1 and 16.D.3 of Mas-Colell et al. (1995). The technical difficulties of bridging discrete and continuous models are overcome by Lemma 2 below which may be of independent interest. In the presentation of the lemma below, the objectives are fixed as $V_{1}, \ldots, V_{n}$, while we allow the initial endowments to vary.

Lemma 2 In the comonotone market under Assumption 2, suppose that $\left(\xi_{1}, \ldots, \xi_{n}\right)$ is proper and $\xi_{i}$ is a continuous function of $X$ on $R(X)$ for each $i \in N,\left\{\xi_{i, k}\right\}_{k=1}^{\infty} \subset \mathcal{X}^{n}$ converges to $\xi_{i}$ uniformly for $i \in N$, and for each $k \in \mathbb{N}, \xi_{1, k}+\cdots+\xi_{n, k} \geq X$ and $\left(\left(X_{1, k}^{*}, \ldots, X_{n, k}^{*}\right), Q^{k}\right)$ is a CCE for the initial endowments $\left(\xi_{1, k}, \ldots, \xi_{n, k}\right)$. Then, there exists a subsequence of $\left\{\left(\left(X_{1, k}^{*}, \ldots, X_{n, k}^{*}\right), Q^{k}\right)\right\}_{k=1}^{\infty}$ which converges to a CCE for the initial endowments $\left(\xi_{1}, \ldots, \xi_{n}\right)$. Here, the convergence is uniform for $\left\{X_{i, k}^{*}\right\}_{k=1}^{\infty}$ and weak for $\left\{Q^{k}\right\}_{k=1}^{\infty}$.

With the help of Lemma 2, we are able to establish the existence and the FTWE results in the RDU-comonotone market.

Theorem 4 In the comonotone market under Assumption 2, the following hold.

(i) If $\left(\xi_{1}, \ldots, \xi_{n}\right)$ is proper and $\xi_{i}$ is a continuous function of $X$ on $R(X)$ for each $i \in N$, then a CCE exists.

(ii) A proper equilibrium (CCE) allocation is necessarily Pareto-optimal.

(iii) A proper Pareto-optimal allocation is necessarily an equilibrium (CCE) allocation for some choice of endowments.

\subsection{An analytical approach for the competitive equilibria}

In general, to find explicit solutions for competitive equilibria in the RDU markets is very challenging. In this section we derive an approach for explicit CCE under some nice conditions on the agents' preferences.

In the following, we shall build a connection from the CCE problem for RDU agents to a UCE problem for expected utility agents with heterogeneous beliefs. Let 
Assumption 2 hold. For $i \in N$, define a probability measure $Q_{i}$ on $(\Omega, \mathcal{B})$ generated by its value on the events $\{X>t\}_{t \in \mathbb{R}}$, specified as

$$
Q_{i}(X>t)=g_{i} \circ \mathbb{P}(X>t), \quad t \in \mathbb{R},
$$

which is indeed a probability measure since $g_{i}(0)=0, g_{i}(1)=1$, and $g_{i}$ is continuous and increasing. Since $g_{i}$ is continuously differentiable, we know that $Q_{i}$ is absolutely continuous with respect to $\mathbb{P}$, and the Radon-Nikodym derivative of $Q_{i}$ with respect to $\mathbb{P}$ is given by ${ }^{12}$

$$
\frac{\mathrm{d} Q_{i}}{\mathrm{~d} \mathbb{P}}=g_{i}^{\prime}\left(S_{X}(X)\right), \quad i \in N
$$

For each $i \in N$ and $Y \in C(X)$,

$$
\begin{aligned}
V_{i}(Y)=R_{g_{i}, u_{i}}(Y)= & \int_{-\infty}^{0}\left(g_{i} \circ \mathbb{P}\left(u_{i}(Y)>z\right)-1\right) \mathrm{d} z \\
& +\int_{0}^{\infty} g_{i} \circ \mathbb{P}\left(u_{i}(Y)>z\right) \mathrm{d} z \\
= & \int_{-\infty}^{0}\left(Q_{i}\left(u_{i}(Y)>z\right)-1\right) \mathrm{d} z \\
& +\int_{0}^{\infty} Q_{i}\left(u_{i}(Y)>z\right) \mathrm{d} z=\mathbb{E}^{Q_{i}}\left[u_{i}(Y)\right] .
\end{aligned}
$$

Note that (10) relies on the useful fact that $(Y, X)$ is comonotonic. Based on (10), in a comonotone market, the individual optimization problem (2) translates to

$$
\max _{X_{i} \in C(X) \cap B\left(\mathbb{Q}, \xi_{i}\right)} \mathbb{E}^{Q_{i}}\left[u_{i}\left(X_{i}\right)\right] .
$$

Now, we introduce the following accessory EU problem in the complete market, for $i \in N$,

$$
\max _{X_{i} \in \mathcal{X} \cap B\left(\mathbb{Q}, \xi_{i}\right)} \mathbb{E}^{Q_{i}}\left[u_{i}\left(X_{i}\right)\right]
$$

Write $V_{i}^{\prime}(Y)=\mathbb{E}^{Q_{i}}\left[u_{i}(Y)\right]$ for $Y \in \mathcal{X}$ and $i \in N$. Since $V_{i}^{\prime}$ and $\mathbb{E}^{\mathbb{Q}}$ are monotone operators, the budget constraint in $B\left(\mathbb{Q}, \xi_{i}\right)$ will be binding at the optimizers.

Clearly, if a solution to (12) is in $C(X)$, then it also solves (11), thus (2). Based on the above observation, we can solve a UCE for the individual objectives $V_{1}^{\prime}, \ldots, V_{n}^{\prime}$. If the resulting UCE is comonotonic, then we arrive at a CCE in the RDU-monotone market as well. Unfortunately, the resulting equilibrium allocation is not necessarily comonotonic in general, and we need some conditions for it to be so.

$\overline{12 \text { Since } \mathcal{B} \text { is generated by } X,(9)}$ holds on $(\Sigma, \mathcal{B})$. 


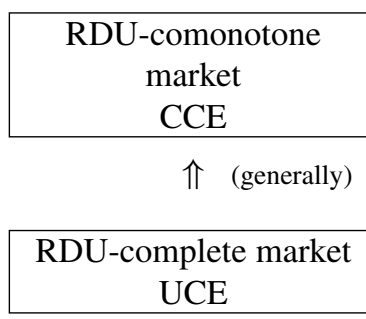

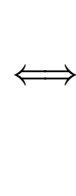

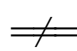

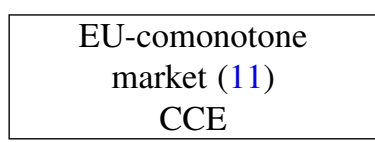

介 (if comonotonic)

\begin{tabular}{c}
\hline EU-complete \\
market (12) \\
UCE \\
\hline
\end{tabular}

(relatively well studied)

First, we try to solve UCE for the individual objectives $V_{1}^{\prime}, \ldots, V_{n}^{\prime}$. Note that $V_{i}^{\prime}$ is not necessarily equal to $V_{i}$ on $\mathcal{X}$, although they are equal on $C(X)$; thus the EU-complete market (12) is not equivalent to an RDU-complete market. Analytical solutions for such a market are available for special classes of utility functions; see e.g. Anthropelos and Kardaras (2017). Below we outline the main steps.

Note that $Q_{1}, \ldots, Q_{n}, \mathbb{P}, \mathbb{Q}$ must all be equivalent probability measures so that (12) has a maximizer. For a fixed $\mathbb{Q} \in \mathcal{P}$, using a Lagrangian method, we obtain a unique solution for (12) under some mild regularity conditions (see e.g. Section 3.1 of Föllmer and Schied (2016) for the technical conditions), that is,

$$
X_{i}=\left(u_{i}^{\prime}\right)^{-1}\left(\frac{\mathrm{d} \mathbb{Q}}{\mathrm{d} Q_{i}} \lambda_{i}\right)
$$

where $\lambda_{i}$ is a positive constant that ensures the binding budget constraint $\mathbb{E}^{\mathbb{Q}}\left[X_{i}\right]=$ $\mathbb{E}^{\mathbb{Q}}\left[\xi_{i}\right]$.

The market clearing condition then boils down to

$$
\sum_{i=1}^{n}\left(u_{i}^{\prime}\right)^{-1}\left(\frac{\mathrm{d} \mathbb{Q}}{\mathrm{d} Q_{i}} \lambda_{i}\right)=X,
$$

and from this equation one can solve for $\mathbb{Q}$. Note that $\lambda_{i}$ also involves $\mathbb{Q}$, making a solution to (14) possibly implicit.

Note that $u_{i}$ is strictly concave, which guarantees that $\left(u_{i}^{\prime}\right)^{-1}$ is a decreasing function. Therefore, $\left(X_{1}, \ldots, X_{n}\right)$ in (13) is comonotonic if $\left(\frac{\mathrm{d} \mathbb{Q}}{\mathrm{d} Q_{1}}, \ldots, \frac{\mathrm{d} \mathbb{Q}}{\mathrm{d} Q_{n}}\right)$ is comonotonic. We summarize our findings in the following proposition.

Proposition 4 Suppose that $\left(\left(X_{1}, \ldots, X_{n}\right), \mathbb{Q}\right)$ is a UCE for the objective functionals $V_{1}^{\prime}, \ldots, V_{n}^{\prime}$. If $\left(\frac{\mathrm{d} \mathbb{Q}}{\mathrm{d} Q_{1}}, \ldots, \frac{\mathrm{d} \mathbb{Q}}{\mathrm{d} Q_{n}}\right)$ is comonotonic, then $\left(\left(X_{1}, \ldots, X_{n}\right), \mathbb{Q}\right)$ is a CCE in the comonotone market under Assumption 2.

A key consequence of Proposition 4 is that the pricing kernel $\eta=\frac{\mathrm{d} \mathbb{Q}}{\mathrm{dP}}$ in a CCE is not necessarily a decreasing function of the total wealth $X$; recall that countercomonotonicity of $(\eta, X)$ is a classic feature of UCE (see Sect. 2.2). To show this, note that, by (9), we have

$$
\eta=\frac{\mathrm{d} \mathbb{Q}}{\mathrm{d} \mathbb{P}}=\frac{\mathrm{d} \mathbb{Q}}{\mathrm{d} Q_{1}} \frac{\mathrm{d} Q_{1}}{\mathrm{dP}}=\frac{\mathrm{d} \mathbb{Q}}{\mathrm{d} Q_{1}} g_{1}^{\prime}\left(S_{X}(X)\right) .
$$


Fig. 1 The pricing kernel $\eta$ as a non-monotone function of $X$ in Example 2

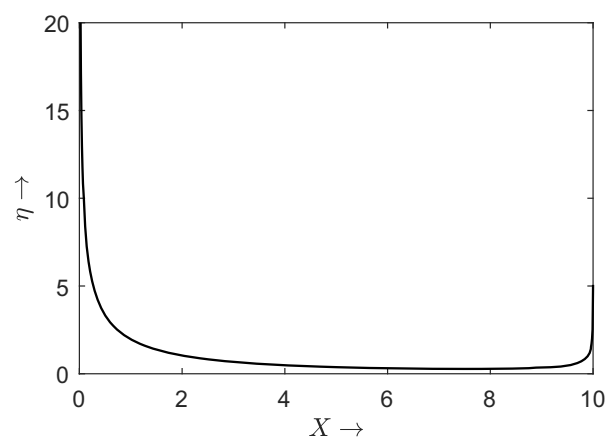

As a result of (14), the term $\frac{d \mathbb{Q}}{\mathrm{d} Q_{1}}$ is a decreasing function of $X$. However, since $g_{1}^{\prime}$ is not necessarily an increasing function unless $g_{1}$ is convex, $g_{1}^{\prime}\left(S_{X}(X)\right)$ is not necessarily a decreasing function of $X$. Indeed, if $g_{i}$ is inverse-S-shaped as in Tversky and Kahneman (1992) (see Fig. 2 in Sect. 6), then $g_{1}^{\prime}$ on $[0,1]$ is first decreasing and then increasing. As a result, $\eta$ as a function of $X$ can be first decreasing and then increasing. This case is shown in Example 2, and here we plot the obtained pricing kernel $\eta$ as a function of $X$ in Fig. 1. Our model is able to accommodate the pricing kernel puzzle (see e.g. Hens and Reichlin 2013), where empirical evidence suggests that the pricing kernel is not a decreasing function of the total wealth $X$, contradicting classic UCE models. Note that a non-decreasing function $\eta$ implies that the comonotonicity constraints in $C(X)$ are binding somewhere in equilibrium; there exists a trade in $\mathcal{X} \backslash C(X)$ that a rational agent would prefer and can afford.

In case that any of $g_{1}, \ldots, g_{n}$ is convex, (15) implies that $\eta$ as a decreasing function of $X$, and hence the above non-classic phenomenon disappears.

We note that Proposition 4 does not tell much if $\left(\frac{\mathrm{d} \mathbb{Q}}{\mathrm{d} Q_{1}}, \ldots, \frac{\mathrm{d} \mathbb{Q}}{\mathrm{d} Q_{n}}\right)$ is not comonotonic. Thus, we are only able to partially solve competitive equilibria for the RDU market under nice conditions guaranteeing the comonotonicity of $\left(\frac{\mathrm{d} \mathbb{Q}}{\mathrm{d} Q_{1}}, \ldots, \frac{\mathrm{d} \mathbb{Q}}{\mathrm{d} Q_{n}}\right)$. A simple example is the case that $g_{1}=\cdots=g_{n}$, and this includes the case of expected utility agents (i.e. $g_{1}, \ldots, g_{n}$ are identity functions). In this case, $Q_{1}=\cdots=Q_{n}$, and obviously $\left(\frac{\mathrm{d} \mathbb{Q}}{\mathrm{d} Q_{1}}, \ldots, \frac{\mathrm{d} \mathbb{Q}}{\mathrm{d} Q_{n}}\right)$ is comonotonic. In fact, this market is closely related to a classic market where the individual preferences are characterized by EU (under the probability measure $Q_{1}=\cdots=Q_{n}$ ) with concave utility functions. In this case, Proposition 4 recovers the result of Borch (1962). See Xia and Zhou (2016) for more recent developments on RDU equilibria with homogeneous probability distortions.

As hinted by the above example, if $g_{1}, \ldots, g_{n}$ are very similar to each other ("almost identical"), then it is more likely that $\left(\frac{\mathrm{d} \mathbb{Q}}{\mathrm{d} Q_{1}}, \ldots, \frac{\mathrm{d} \mathbb{Q}}{\mathrm{d} Q_{n}}\right)$ is comonotonic. In Sect. 4.3, we formalize this concept for the case of exponential utility functions.

\subsection{The RDU-exponential market}

In this section, we focus on the case where the utility functions $u_{1}, \ldots, u_{n}$ are exponential. 


\section{Assumption 3 Let}

$$
u_{i}(x)=-\exp \left(-\frac{x}{\theta_{i}}\right), x \in \mathbb{R}
$$

for $i \in N$, where $\theta_{1}, \ldots, \theta_{n}>0$ are parameters representing risk tolerance.

From (13), the problem (12) has a unique solution

$$
X_{i}=-\theta_{i} \ln \left(\theta_{i} \lambda_{i} \frac{\mathrm{d} \mathbb{Q}}{\mathrm{d} Q_{i}}\right)
$$

To determine the coefficient $\lambda_{i}$ from $\mathbb{E}^{\mathbb{Q}}\left[\xi_{i}\right]=\mathbb{E}^{\mathbb{Q}}\left[X_{i}\right]$, we have

$$
\mathbb{E}^{\mathbb{Q}}\left[\xi_{i}\right]=-\theta_{i}\left(\mathbb{E}^{\mathbb{Q}}\left[\frac{\mathrm{d} \mathbb{Q}}{\mathrm{d} Q_{i}}\right]+\ln \left(\theta_{i} \lambda_{i}\right)\right)=-\theta_{i}\left(D_{K L}\left(\mathbb{Q} \| Q_{i}\right)+\ln \left(\theta_{i} \lambda_{i}\right)\right),
$$

where $D_{K L}\left(\mathbb{Q} \| Q_{i}\right)=\mathbb{E}^{\mathbb{Q}}\left[\frac{\mathrm{d} \mathbb{Q}}{\mathrm{d} Q_{i}}\right]$ is the Kullback-Leibler divergence from $Q_{i}$ to $\mathbb{Q}$. Then,

$$
X_{i}=\theta_{i} \ln \left(\frac{\mathrm{d} Q_{i}}{\mathrm{~d} \mathbb{Q}}\right)+\theta_{i} D_{K L}\left(\mathbb{Q} \| Q_{i}\right)+\mathbb{E}^{\mathbb{Q}}\left[\xi_{i}\right]=\theta_{i} \ln \left(\frac{\mathrm{d} Q_{i}}{\mathrm{~d} \mathbb{Q}}\right)+c_{i},
$$

for $c_{i} \in \mathbb{R}$. The market clearing constraint implies that, for each $j=1, \ldots, n$,

$$
X=\sum_{i=1}^{n} X_{i}=\sum_{i=1}^{n} \theta_{i} \ln \left(\frac{\mathrm{d} Q_{i}}{\mathrm{~d} \mathbb{Q}}\right)+\sum_{i=1}^{n} c_{i}=\bar{\theta} \ln \left(\frac{\mathrm{d} \mathbb{P}}{\mathrm{d} \mathbb{Q}}\right)+\sum_{i=1}^{n} \theta_{i} \ln \left(\frac{\mathrm{d} Q_{i}}{\mathrm{~d} \mathbb{P}}\right)+\bar{c}
$$

where $\bar{\theta}=\sum_{i=1}^{n} \theta_{i}$ and $\bar{c}=\sum_{i=1}^{n} c_{i}$. Solving the above equation we arrive at

$$
\frac{\mathrm{d} \mathbb{Q}}{\mathrm{d} \mathbb{P}}=\exp \left\{\frac{1}{\bar{\theta}}\left(\sum_{i=1}^{n} \theta_{i} \ln \left(\frac{\mathrm{d} Q_{i}}{\mathrm{dP}}\right)+\bar{c}-X\right)\right\}
$$

Meanwhile,

$$
X=\sum_{i=1}^{n} X_{i}=\sum_{i=1}^{n} \theta_{i} \ln \left(\frac{\mathrm{d} Q_{i}}{\mathrm{~d} \mathbb{Q}}\right)+\sum_{i=1}^{n} c_{i}=\bar{\theta} \ln \left(\frac{\mathrm{d} Q_{j}}{\mathrm{~d} \mathbb{Q}}\right)+\sum_{i=1}^{n} \theta_{i} \ln \left(\frac{\mathrm{d} Q_{i}}{\mathrm{~d} Q_{j}}\right)+\bar{c}
$$

Thus, for the $j$ th agent, the optimal risk allocation for (12) is

$$
X_{j}=\frac{\theta_{j}}{\bar{\theta}}\left(X-\sum_{i=1}^{n} \theta_{i} \ln \left(\frac{\mathrm{d} Q_{i}}{\mathrm{~d} Q_{j}}\right)-\bar{c}\right)+c_{j}=\frac{\theta_{j}}{\bar{\theta}}\left(\phi_{j}(X)-\bar{c}\right)+c_{j}
$$


where

$$
\phi_{j}(X)=X-\sum_{i=1}^{n} \theta_{i} \ln \left(\frac{\mathrm{d} Q_{i}}{\mathrm{~d} Q_{j}}\right)
$$

Next, we check if this risk allocation also solves (2), i.e., we check the comonotonicity constraint $X_{j} \in C(X)$. This is equivalent to $\frac{\theta_{j}}{\bar{\theta}}\left(X-\sum_{i=1}^{n} \theta_{i} \ln \left(\frac{\mathrm{d} Q_{i}}{\mathrm{~d} Q_{j}}\right)\right) \in C(X)$, or $\phi_{j}^{\prime}(x) \geq 0$ for all $j=1, \ldots, n$ and $x \in R(X)$. This is a sufficient condition for the existence of the solution to Problem (2). Let $q_{i}(x)=\frac{\mathrm{d} Q_{i}(X \leq x)}{\mathrm{d} x}$ for $i=1, \ldots, n$. Then, the condition $\phi_{j}^{\prime}(x) \geq 0$ for all $j=1, \ldots, n$ and $x \in R(X)$ is equivalent to

$$
\bar{\theta}^{-1}+\frac{q_{j}^{\prime}(x)}{q_{j}(x)} \geq \sum_{i=1}^{n} \frac{\theta_{i}}{\bar{\theta}} \frac{q_{i}^{\prime}(x)}{q_{i}(x)}, \quad \text { for all } j=1, \ldots, n \text { and } x \in R(X),
$$

or

$$
\inf _{x \in \mathbb{R}} \inf _{j=1, \ldots, n}\left\{\bar{\theta}^{-1}+\frac{q_{j}^{\prime}(x)}{q_{j}(x)}-\sum_{i=1}^{n} \frac{\theta_{i}}{\bar{\theta}} \frac{q_{i}^{\prime}(x)}{q_{i}(x)}\right\} \geq 0 .
$$

This result is summarized in the following theorem.

Theorem 5 In the comonotone market under Assumptions 2 and 3, if (20) holds, then a CCE is given by $\left(\left(X_{1}, \ldots, X_{n}\right), \mathbb{Q}\right)$ in $(17)-(18)$.

Condition (20) has the following intuition. The term $\frac{q_{j}^{\prime}(x)}{q_{j}(x)}-\sum_{i=1}^{n} \frac{\theta_{i}}{\bar{\theta}} \frac{q_{i}^{\prime}(x)}{q_{i}(x)}$ states the level of ambiguity aversion for agent $j$ relative to a weighted average of that of other agents, and $\bar{\theta}^{-1}$ is a measure of risk aversion for all agents in the market. Roughly speaking, if the overall risk aversion is larger than the ambiguity aversion of each agent relative to others, then the complete market yields also an equilibrium in the comonotone market, similarly to the case of a classic expected utility market. The case of power utilities is presented in Appendix A.1.

\section{An algorithm to compute the competitive equilibria}

Generally, explicit forms of the competitive equilibria for the RDU-comonotone market are not available except for the special cases studied in Sects. 4.2-4.3. In this section, we propose an algorithm to numerically obtain competitive equilibrium based on discretization, which works for both the DU-comonotone market and the RDUcomonotone market. Its numerical performance is reported in Sect. 6.

We assume the same assumptions as in Theorem 4 to guarantee the existence of a CCE. Without loss of generality, take $R(X)=[0, \bar{X}]$, where $\bar{X}<\infty$, for the purpose of the algorithm. First, choose $m \in \mathbb{Z}^{+}$such that $\varepsilon=\bar{X} / m$ is small, and approximate $X$ by a discrete random variable $\hat{X}$ which takes value in a finite set $R(\hat{X}):=\left\{x_{k}=k \varepsilon\right.$ : 
$k=1, \ldots, m\}$. Then, we propose the algorithm of the discretization approach on the discrete probability space $(R(\hat{X}), \mathcal{B}(R(\hat{X})), \hat{\mathbb{P}})$, where $\mathcal{B}(R(\hat{X}))$ is the Borel $\sigma$-algebra of $R(\hat{X})$ and $\hat{\mathbb{P}}\left(\left\{x_{k}\right\}\right)=\mathbb{P}((k-1) \varepsilon<X \leq k \varepsilon)$ for $k=1, \ldots, m$. The discretized version of the initial endowment for agent $\bar{i}$, denoted by $\hat{\xi}_{i}$, satisfies $\hat{\xi}_{i}\left(x_{k}\right)=\xi_{i}\left(x_{k}\right)$ for $k=1, \ldots, m$. From now on, we work with discrete random variables $\hat{X}$ and $\hat{\xi}_{1}, \ldots, \hat{\xi}_{n}$, and study this discrete market $(R(\hat{X}), \mathcal{B}(R(\hat{X})), \hat{\mathbb{P}})$ as an approximation to the original market.

Suppose the algorithm starts with an initial pricing measure $\hat{Q}_{0}=\left(q_{0, k}\right)_{k=1, \ldots, m}$, which is the initial guess of price $q_{0, k}:=\hat{Q}_{0}\left(\hat{X} \geq x_{k}\right)$, and initial allocation functions $\psi_{0}^{i}, i \in N$ such that $\left(\psi_{0}^{i}(\hat{X}), \hat{X}-\psi_{0}^{i}(\hat{X})\right)$ is comonotonic, $i \in N$. Without prior knowledge, we may choose the initial values as $\hat{Q}_{0}=\hat{\mathbb{P}}$ and $\psi_{0}^{i}(\hat{X})=\hat{\xi}_{i}$, assuming $\left(\xi_{1}, \ldots, \xi_{n}\right) \in \mathbb{A}_{n}^{c}(X)$.

Remark 1 Our idea is to use a multi-step discrete procedure to mimic the actual one-period trading (which leads to an equilibrium). In order to make sense of the comonotone market, we have to assume that the individual endowments are comonotonic at each step of the trading. If the initial endowments are already comonotonic, then this is not a problem, and we set $\psi_{0}^{i}(\hat{X})=\hat{\xi}_{i}, i \in N$ as mentioned above. If $\left(\xi_{1}, \ldots, \xi_{n}\right) \notin \mathbb{A}_{n}^{c}(X)$, i.e. the initial endowments are not comonotonic, then we need to first transform it into another initial condition in which endowments are comonotonic. For this purpose, we may use

$$
\psi_{0}^{i}(\hat{X})=\frac{\mathbb{E}^{\hat{Q}_{0}}\left[\hat{\xi_{i}}\right]}{\mathbb{E}^{\hat{Q}_{0}}[\hat{X}]} \hat{X}
$$

as the initial endowment for agent $i$. This interprets into the situation where each agent liquidates his or her initial endowment and trade it for portion of the total endowment under the price $\hat{Q}_{0}$, before the actual trading starts.

Due to Lemma 1, for each $i \in N$, we aim to find a non-negative allocation $\left(Y_{1}, \ldots, Y_{n}\right) \in \mathbb{A}_{n}^{c}(\hat{X})$ such that $V_{i}\left(Y_{i}-\mathbb{E}^{\mathbb{Q}}\left[Y_{i}\right]+\mathbb{E}^{\mathbb{Q}}\left[\hat{\xi}_{i}\right]\right)$ is maximized. Therefore, we define a "score function" of agent $i$ as

$$
v_{i}\left(Y_{i} ; Q\right)=V_{i}\left(Y_{i}-\mathbb{E}^{Q}\left[Y_{i}\right]+\mathbb{E}^{Q}\left[\hat{\xi}_{i}\right]\right),
$$

for $Y_{i} \in C(\hat{X})$ and $Q \in \mathcal{P}$.

Let $\delta_{0,1}^{i}=\psi_{0}^{i}\left(x_{1}\right)$ and $\delta_{0, k}^{i}=\psi_{0}^{i}\left(x_{k}\right)-\psi_{0}^{i}\left(x_{k-1}\right)$ for $k=2, \ldots, m$. Since $\left(\psi_{0}^{i}(\hat{X}), \hat{X}-\psi_{0}^{i}(\hat{X})\right)$ is comonotonic, we have $\delta_{0, k}^{i} \in[0, \varepsilon]$ for $k=1, \ldots, m$. For any $x \in[0, \bar{X}]$, it holds that

$$
\psi_{0}^{i}(x)=\sum_{k=1}^{m} \delta_{0, k}^{i} \mathbb{I}_{\left\{x \geq x_{k}\right\}} .
$$

Our target is to recursively update $\delta_{0, k}^{i}$ and $q_{0, k}$ simultaneously. After the $(j-1)$ th step, $j=1,2, \ldots, m-1$, the pricing measure is denoted by $\hat{Q}_{j-1}=\left(q_{j-1, k}\right)_{k=1, \ldots, m}$, 
and the allocation function for agent $i$, denoted by $\psi_{j-1}^{i}$, becomes $\psi_{j-1}^{i}(x)=$ $\sum_{k=1}^{m} \delta_{j-1, k}^{i} \mathbb{I}_{\left\{x \geq x_{k}\right\}}$. In the $j$ th step, we want to replace $\delta_{j-1, j}^{i}$ by $\delta_{j, j}^{i}$ such that the agent's utility is improved. Denote $\psi_{j}^{i}(x)=\sum_{k=1}^{m} \delta_{j, k}^{i} \mathbb{I}_{\left\{x \geq x_{k}\right\}}$, where we keep $\delta_{j, k}^{i}=\delta_{j-1, k}^{i}$ for $k \neq j$. Note that $\psi_{j}^{i}(x)-\psi_{j-1}^{i}(x)=\left(\delta_{j, j}^{i}-\delta_{j-1, j}^{i}\right) \mathbb{I}_{\left\{x \geq x_{j}\right\}}$. Since $\varepsilon$ is small, the difference between agent $i$ 's utilities for $\psi_{j-1}^{i}$ and $\psi_{j}^{i}$, assuming the pricing measure is $\hat{Q}_{j-1}$, can be approximated by using a first-order Taylor's expansion as follows:

$$
\begin{aligned}
& v_{i}\left(\psi_{j}^{i}(\hat{X}) ; \hat{Q}_{j-1}\right)-v_{i}\left(\psi_{j-1}^{i}(\hat{X}) ; \hat{Q}_{j-1}\right) \\
& =\mathbb{E}^{Q_{i}}\left[u _ { i } \left(\psi_{j}^{i}(\hat{X})-\mathbb{E}^{\hat{Q}_{j-1}}\left[\psi_{j}^{i}(\hat{X})\right]+\mathbb{E}^{\left.\left.\hat{Q}_{j-1}\left[\hat{\xi}_{i}\right]\right)\right]}\right.\right.
\end{aligned}
$$

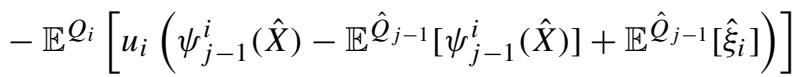

$$
\begin{aligned}
& \approx \mathbb{E}^{Q_{i}}\left[u_{i}^{\prime}\left(\eta_{j-1}^{i}\right)\left(\mathbb{I}_{\left\{\hat{X} \geq x_{j}\right\}}-q_{j-1, j}\right)\right]\left(\delta_{j, j}^{i}-\delta_{j-1, j}^{i}\right) \\
& =\mathbb{E}^{Q_{i}}\left[u_{i}^{\prime}\left(\eta_{j-1}^{i}\right)\right]\left(r_{j-1}^{i}-q_{j-1, j}\right)\left(\delta_{j, j}^{i}-\delta_{j-1, j}^{i}\right),
\end{aligned}
$$

where

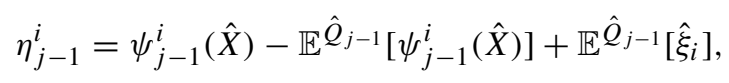

and

$$
r_{j-1}^{i}=\frac{\mathbb{E}^{Q_{i}}\left[u_{i}^{\prime}\left(\eta_{j-1}^{i}\right) \mathbb{I}_{\left\{\hat{X} \geq x_{j}\right\}}\right]}{\mathbb{E}^{Q_{i}}\left[u_{i}^{\prime}\left(\eta_{j-1}^{i}\right)\right]}
$$

In (21), $\delta_{j, j}^{i}-\delta_{j-1, j}^{i}$ represents the position change of the allocation function at value $x_{j}$ for agent $i$ at the $j$ th step of the algorithm. Note that if each agent updates $\delta_{j, j}^{i}, i \in N$ according to (21), then $\sum_{i=1}^{n} \delta_{j, j}^{i}=\varepsilon$ does not necessarily hold, namely, the market is not cleared. As the agents start to adjust their positions, the pricing measure updates simultaneously, so that the market clearing condition is satisfied, i.e. $q_{j-1, j}$ is replaced by $q_{j, j}$ in (21) so that $\sum_{i=1}^{n} \delta_{j, j}^{i}=\varepsilon$. To make this happen, let $\hat{Q}_{j}=\left(q_{j, k}\right)_{k=1, \ldots, m}$ with $q_{j, k}=q_{j-1, k}$ for $k \neq j$ and

$$
q_{j, j}=\frac{1}{2}\left(\operatorname{sl}_{i}\left\{r_{j-1}^{i}\right\}+\max _{i}\left\{r_{j-1}^{i}\right\}\right),
$$

where $\operatorname{sl}_{i}\left\{r_{j-1}^{i}\right\}$ is the second largest value among $r_{j-1}^{i}, i \in N$. Write

$$
D_{j}^{i}=\mathbb{E}^{Q_{i}}\left[u_{i}^{\prime}\left(\eta_{j-1}^{i}\right)\left(\mathbb{I}_{\left\{\hat{X} \geq x_{j}\right\}}-q_{j, j}\right)\right]=\mathbb{E}^{Q_{i}}\left[u_{i}^{\prime}\left(\eta_{j-1}^{i}\right)\right]\left(r_{j-1}^{i}-q_{j, j}\right) .
$$


We shall update $\delta_{j, j}^{i}, i \in N$ such that $D_{j}^{i}\left(\delta_{j, j}^{i}-\delta_{j-1, j}^{i}\right)$ is maximized instead of (21). Clearly, at most one of $D_{j}^{i}$ for $i \in N$ is positive, and at least one of them is nonnegative. For agent $i, i \in N$, he/she should set $\delta_{j, j}^{i}=\varepsilon$ if $D_{j}^{i}>0$ (or equivalently $r_{j-1}^{i}-q_{j, j}>0$ ) and $\delta_{j, j}^{i}=0$ if $D_{j}^{i}<0$. If $D_{j}^{i}=0$ and $\max _{i}\left\{D_{j}^{i}\right\}>0$, then $\delta_{j, j}^{i}=0$, and if $D_{j}^{i}=0$ and $\max _{i}\left\{D_{j}^{i}\right\}=0$, then $\delta_{j, j}^{i}$ could be any number in $[0, \varepsilon]$ summing up to $\varepsilon$. For convenience, and to summarize the above cases, we set

$$
\delta_{j, j}^{i}=\frac{\varepsilon \mathbb{I}_{\left\{\max _{i}\left\{D_{j}^{i}\right\}=0\right\}}}{\#\left\{D_{j}^{i}=0, i=1, \ldots, n\right\}} \quad \text { if } D_{j}^{i}=0,
$$

where $\# A$ is the cardinality of the set $A$. In this way, $\sum_{i=1}^{n} \delta_{j, j}^{i}=\varepsilon$, and the market clearing condition is satisfied. Indeed, $q_{j, j}$ may be chosen at any value in the interval $\left[\mathrm{sl}_{i}\left\{r_{j-1}^{i}\right\}, \max _{i}\left\{r_{j-1}^{i}\right\}\right]$, and we choose (24) for convenience.

Now, since we have updated $\hat{Q}_{j}$ and $\psi_{j}^{i}$ for all $i$, we can determine the function $\eta_{j}^{i}$, and proceed to step $j+1$. We repeat this procedure $m$ times so that the values of the allocation functions and the pricing measure at each $x_{k}, k=1, \ldots, m$ is updated once.

Finally, we set the resulting allocation functions and the pricing measure from the above procedure as new initial values, and run the algorithm above again, until a satisfactory convergence is achieved. We summarize the entire algorithm below.

\section{Algorithm.}

(1) $\operatorname{Set} \psi_{0}^{i}(\hat{X})=\hat{\xi}_{i}$ as the initial endowment for agent $i$ for all $i=1, \ldots, n$, and $q_{0, k}=\hat{\mathbb{P}}\left(\hat{X} \geq x_{k}\right), k=1, \ldots, m$, as the initial pricing measure.

(2) At the jth step, $j=1,2, \ldots$,

(a) Determine $r_{j-1}^{i}$ for $i=1, \ldots, n$ from (23).

(b) Set $q_{j, k}=q_{j-1, k}$ for $k \neq j$ and $q_{j, j}=\frac{1}{2}\left(\operatorname{sl}_{i}\left\{r_{j-1}^{i}\right\}+\max _{i}\left\{r_{j-1}^{i}\right\}\right)$.

(c) Determine $D_{j}^{i}$ for $i=1, \ldots, n$ from $(25)$.

(d) For $i=1, \ldots, n$, set

$$
\delta_{j, k}^{i}=\delta_{j-1, k}^{i}, \text { for } k \neq j, \quad \text { and } \delta_{j, j}^{i}= \begin{cases}\varepsilon, & \text { if } D_{j}^{i}>0, \\ 0, & \text { if } D_{j}^{i}<0 \\ \varepsilon \frac{\mathbb{I}_{\left\{\max _{i}\left\{D_{j}^{i}\right\}=0\right\}},}{\#\left\{D_{j}^{i}=0, i=1, \ldots, n\right\}}, & \text { if } D_{j}^{i}=0 .\end{cases}
$$

(e) Determine $\eta_{j}^{i}$, for $i=1, \ldots, n$ from (22).

(3) Run (2) for $j=1, \ldots, m$. After $m$ steps, the wealth allocation function for agent $i$ becomes $\psi_{m}^{i}(\hat{X})=\sum_{k=1}^{m} \delta_{m, k}^{i} \mathbb{I}_{\left\{\hat{X} \geq x_{k}\right\}}$, and the pricing probability is $\hat{Q}_{m}=$ $\left(q_{m, k}\right)_{k=1, \ldots, m}$. 
(4) Reset the values $\hat{Q}_{0}=\hat{Q}_{m}$, and $\psi_{0}^{i}=\psi_{m}^{i}+\mathbb{E}^{\hat{Q}_{0}}\left[\hat{\xi}_{i}-\psi_{m}^{i}(\hat{X})\right]$ or all $i=1, \ldots, n$, and repeat the process (2)-(3) until results converge. The outputs are the final values of $\psi_{m}^{i}, i=1, \ldots, n$ and $\hat{Q}_{m}$.

Remark 2 The aforementioned flexibility of the choice of $q_{j, j}$ in (2-b) will affect largely the resulting equilibrium price in cases where the equilibrium price is not unique, for instance, the case of the DU market, as established in Theorem 1. In cases where the equilibrium price is unique (e.g. the setting of Theorem 5), this choice has a minor influence on the resulting equilibrium price in our numerical experiments of Sect. 6.

Due to technical challenges, for general RDU-markets, convergence and termination conditions of the algorithm are still unclear. Nevertheless, we can show that in a DU-comonotone market, the algorithm converges to a CCE. This result is included in Proposition 5 in Appendix A.3 for the interested reader.

\section{Illustration of the algorithm}

In this section, we illustrate the algorithm introduced in Sect. 5 by means of four examples. In the first three examples, we show that the algorithm works quite well in cases where we know the equilibrium exactly. Thereafter, we provide another example where the equilibrium solution is not known exactly.

We use certainty equivalents (CEQ) to assess the quality of our algorithm and to evaluate the improvement from prior to posterior allocations. For $i \in N$, let $\mathrm{CEQ}_{i}^{\text {prior }}$ and $\mathrm{CEQ}_{i}^{\text {post }}$ be constants such that

$$
V_{i}\left(\mathrm{CEQ}_{i}^{\text {prior }}\right)=V_{i}\left(\xi_{i}\right) \text { and } V_{i}\left(\mathrm{CEQ}_{i}^{\text {post }}\right)=V_{i}\left(X_{i}\right)
$$

where $\left(X_{1}, \ldots, X_{n}\right)$ is an equilibrium allocation, either obtained via analytical results or arrived from our algorithm.

We parameterize the distortion function $g$ as in Tversky and Kahneman (1992) for $\gamma \in(0,1)$ :

$$
g(s ; \gamma)=\frac{s^{\gamma}}{\left(s^{\gamma}+(1-s)^{\gamma}\right)^{1 / \gamma}}, \quad s \in[0,1] .
$$

Rieger and Wang (2006) show that $g(\cdot ; \gamma)$ in (27) is increasing and inverse- $S$ shaped for $\gamma \in(0.279,1)$. We display this function in Fig. 2 for three choices of $\gamma$. For larger parameters $\gamma$, we have that the distortion function is closer to the identity function. Therefore, we say that distortion functions with a smaller $\gamma$ is more distorted.

For illustrative purposes, throughout this section, we assume that $\varepsilon=0.01, m=$ 1000 , and $\hat{\mathbb{P}}$ is the discrete uniform distribution over $\{k \varepsilon: k=1, \ldots, m\}$. Moreover, we have three agents with $\xi_{i}=\frac{1}{3} X$ for $i=1,2,3$.

Example 1 (Dual utilities) Let $N=\{1,2,3\}$, and the three agents use dual utilities with distortion functions $g_{i}(\cdot)=g\left(\cdot ; \gamma_{i}\right)$, where $\gamma_{1}=0.4, \gamma_{2}=0.6$, and $\gamma_{3}=0.8$. 
Fig. 2 Three examples of $g(\cdot ; \gamma)$ in $(27)$, for $\gamma=0.4,0.6,0.8$

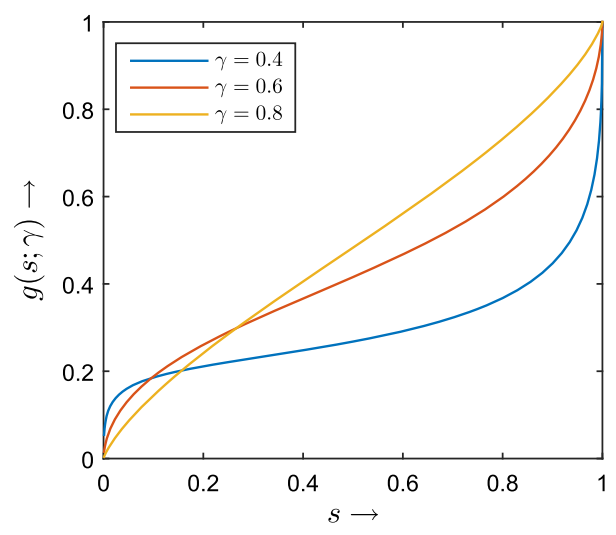

Figure 3 plots an equilibrium (CCE) price and allocation obtained from the algorithm. The equilibrium (CCE) price is reported in terms of $\mathbb{Q}\left(X \geq S_{X}^{-1}(s)\right)$, where $S_{X}^{-1}$ is the inverse function of $S_{X}$, so that it can be compared with the distortion functions via the relation (8). Since $u_{i}^{\prime}(\cdot)=1$, the algorithm is unaffected by the initial values, and so it yields the same solution after every loop. The equilibrium (CCE) price critically depends on our choice to select $q_{j, j}$ as in (24).

In Fig. 3, we see that the price measure is indeed between $g_{N, 1}$ and $g_{N, 2}$, as shown in Theorem 1. Also, the allocations are exactly as shown in Proposition 1 . The equilibrium (CCE) allocations exhibit a regime-switching at $X \approx 7.3$ and $X \approx 9.0$, which coincide with the crossing of the distortion functions at $s \approx S_{X}(7.3)=0.27$ and $s \approx S_{X}(9.0)=$ 0.1 . Note that the pricing kernels, as characterized in Theorem 1 , are all not countercomonotone with $X$. Therefore, no UCEs exist in this example.

Table 1 shows the welfare gains. We find that Agents 1 and 2 also gain in a CCE, even though these agent bear little risk. In particular, Agent 1 mitigates his or her risk substantially, because it has the most distorted preferences.

Example 2 (Rank-dependent utilities with explicit solution 1)

In this example, we want to compare our algorithm with a case where we know an exact solution. Let $N=\{1,2,3\}$, and the three agents use an exponential utility function with risk tolerance parameters $\theta_{1}=3, \theta_{2}=2$ and $\theta_{3}=1$. Moreover, the agents use the distortion function $g_{i}(\cdot)=g(\cdot ; \gamma)$, with $\gamma=0.6$.

If the distortions are the same for every agent, the preferences are represented to expected utilities, but with an alternative probability measure of $X$. This holds true because we assume that admissible allocations are comonotonic with the total endowments. Since the CCE is Pareto-optimal (Theorem 4), and any Pareto-optimal risk allocation with exponential utilities is proportional to $X$ (Wilson 1968), we find in Fig. 4 that the risk allocation in a CCE is proportional to $X$. The algorithm selects very closely the equilibrium solution. This CCE is not a UCE in the RDU-complete market, since the pricing kernel is not counter-comonotone with $X$.

We find that the allocation for Agent $2, X_{2}$, is very close to the initial endowment $\xi_{2}$. Therefore, Agent 2 does not benefit significantly in the equilibrium, as confirmed 


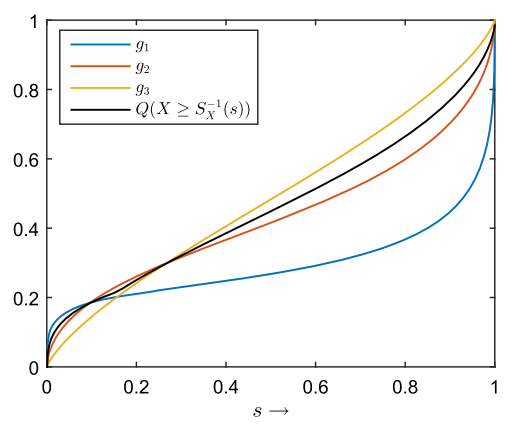

(a) Distortion functions and equilibrium price (exact)

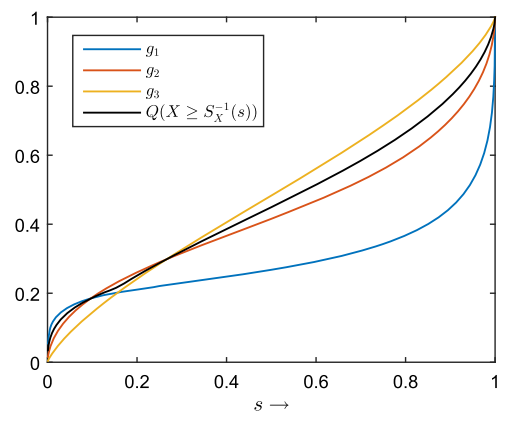

(b) Distortion functions and equilibrium price (algorithm)

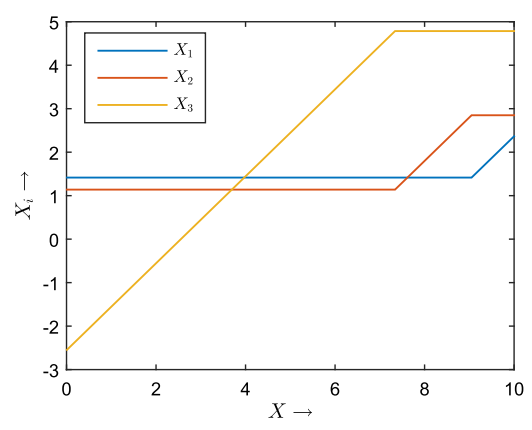

(d) Equilibrium allocation (algorithm)

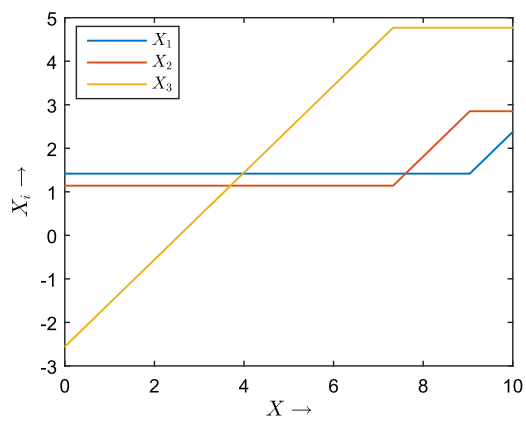

(c) Equilibrium allocation (exact)

Fig. 3 A CCE corresponding to Example 1. In the top figures, the black line is the price $\mathbb{Q}\left(X \geq S_{X}^{-1}(s)\right)$ (we display $\mathbb{Q}$ as $Q$ ), both exact and obtained from the algorithm. The other lines are the distortion functions $g_{1}, g_{2}$ and $g_{3}$. The bottom figures show the corresponding equilibrium (CCE) allocations $X_{1}, X_{2}$ and $X_{3}$, both exact and obtained from the algorithm

Table 1 The certainty equivalents before and after risk sharing, corresponding to Example 1

\begin{tabular}{|c|c|c|c|c|}
\hline & $\mathrm{CEQ}_{i}^{\text {prior }}$ & $\mathrm{CEQ}_{i}^{\text {post }}$ (theoretical) & $\%$ increase & $\mathrm{CEQ}_{i}^{\text {post }}$ (algorithm) \\
\hline Agent 1 & 0.99 & 1.56 & 58.0 & 1.56 \\
\hline Agent 2 & 1.44 & 1.56 & 8.3 & 1.56 \\
\hline Agent 3 & 1.63 & 1.86 & 14.7 & 1.86 \\
\hline
\end{tabular}

in Table 2. Table 2 also confirms that the algorithm selects an equilibrium that is close to the theoretical equilibrium.

Example 3 (Rank-dependent utilities with explicit solution 2) Let $N=\{1,2,3\}$, and the three agents use an exponential utility function with parameters $\theta_{1}=2, \theta_{2}=1.5$, and $\theta_{3}=1$. In order for the condition in Theorem 5 to hold, the derivatives of the distortion functions need to be close to each other uniformly. Therefore, we choose, for $i=1,2,3$ and $\delta>0$ : 


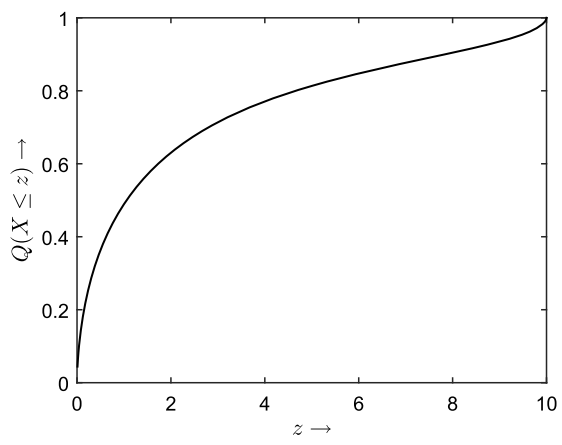

(a) Equilibrium price (exact)

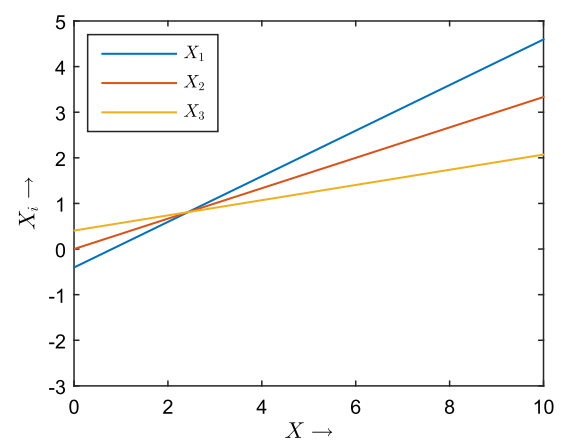

(c) Equilibrium allocation (exact)

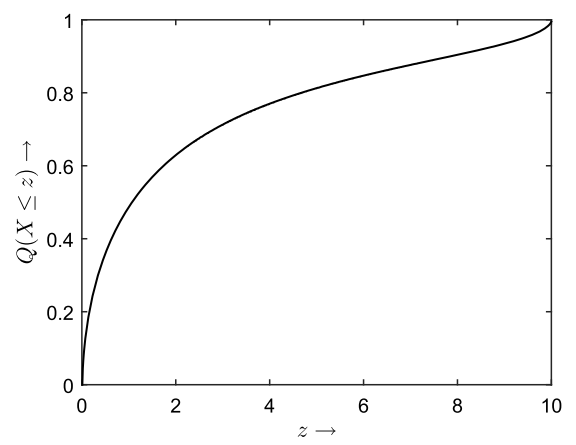

(b) Equilibrium price (algorithm)

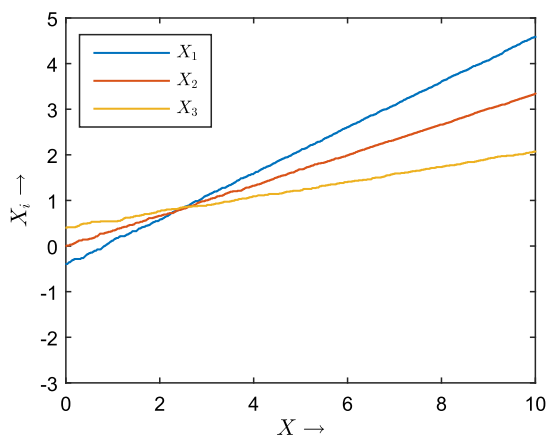

(d) Equilibrium allocation (algorithm)

Fig. 4 The exact and approximated equilibrium (CCE) solution corresponding to Example 2. The top figures display $\mathbb{Q}(X \leq z)$ (we display $\mathbb{Q}$ as $Q$ ), both exact and obtained from the algorithm. The bottom figures display the allocation both exact and obtained from the algorithm

Table 2 The certainty equivalents before and after risk sharing, corresponding to Example 2

\begin{tabular}{lllcc}
\hline & $\mathrm{CEQ}_{i}^{\text {prior }}$ & $\mathrm{CEQ}_{i}^{\text {post }}$ (theoretical) & \% increase & $\mathrm{CEQ}_{i}^{\text {post }}$ (algorithm) \\
\hline Agent 1 & 1.21 & 1.25 & 3.6 & 1.25 \\
Agent 2 & 1.10 & 1.10 & 0.0 & 1.10 \\
Agent 3 & 0.86 & 0.96 & 11.3 & 0.96 \\
\hline
\end{tabular}

$$
g_{i}(s)=a g\left(\frac{s+\delta}{1+2 \delta} ; \gamma_{i}\right)+b, \quad s \in[0,1]
$$

where $a, b$ are constants such that $g_{i}(0)=1-g_{i}(1)=0$. Fix $\delta=0.05, \gamma_{1}=0.55$, $\gamma_{2}=0.6$, and $\gamma_{3}=0.65$. We display the distortion functions in Fig. 5 .

In Fig. 6, we find that the algorithm selects again very closely an equilibrium (CCE) solution. Moreover, in Table 3 we show the welfare gains in the risk allocation, which also shows that the algorithm performs well in selecting an equilibrium solution. 
Fig. 5 Three examples of $g_{i}$ in (28), for $\delta=0.05$ and $\gamma=0.55,0.6,0.65$

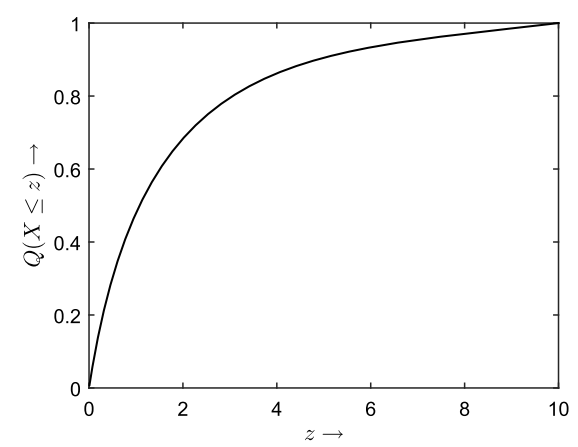

(a) Equilibrium price (exact)

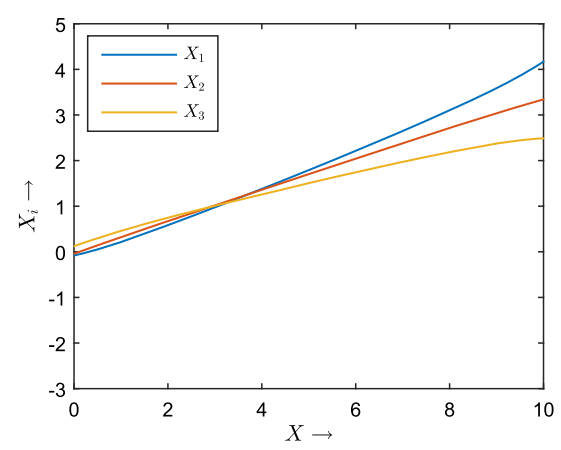

(c) Equilibrium allocation (exact)
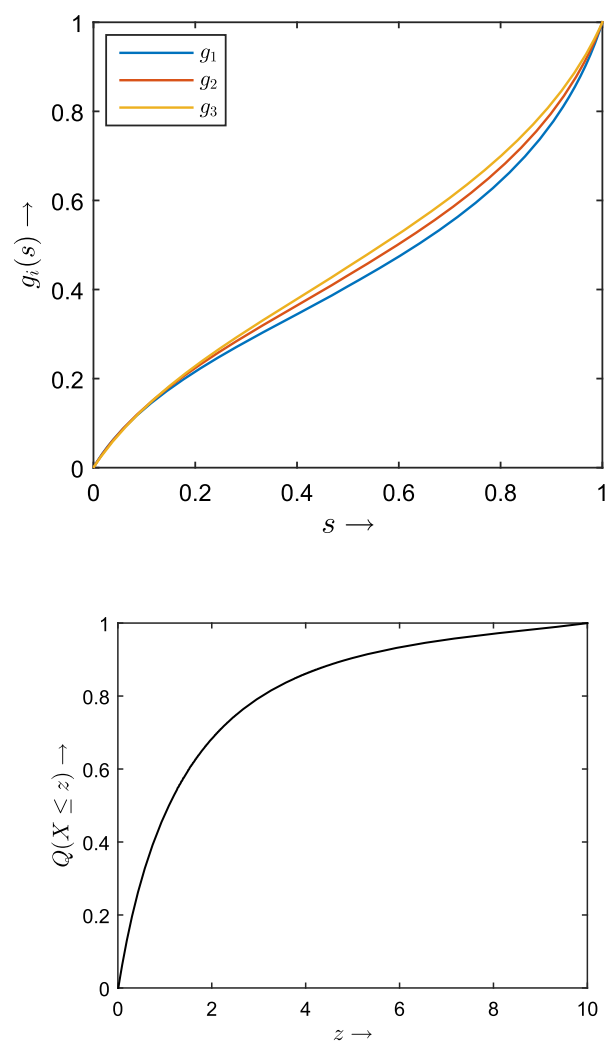

(b) Equilibrium price (algorithm)

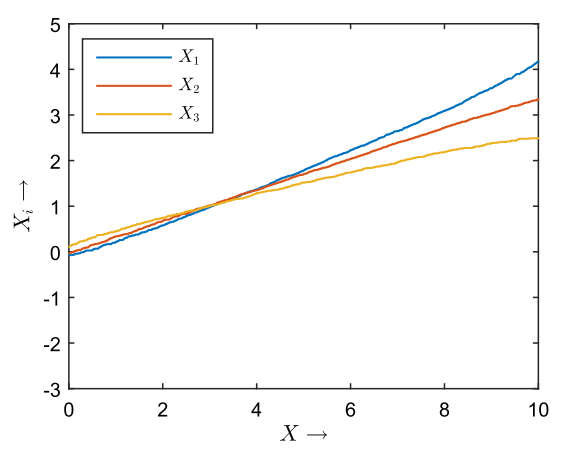

(d) Equilibrium allocation (algorithm)

Fig. 6 The exact and approximated equilibrium (CCE) solution corresponding to Example 3. The top figures display $\mathbb{Q}(X \leq z)$ (we display $\mathbb{Q}$ as $Q$ ), both exact and obtained from the algorithm. The bottom figures display the allocation both exact and obtained from the algorithm 
Table 3 The certainty equivalents before and after risk sharing, corresponding to Example 3

\begin{tabular}{lllll}
\hline & $\mathrm{CEQ}_{i}^{\text {prior }}$ & $\mathrm{CEQ}_{i}^{\text {post }}$ (theoretical) & \% increase & $\mathrm{CEQ}_{i}^{\text {post }}$ (algorithm) \\
\hline Agent 1 & 1.156 & 1.167 & 0.9 & 1.167 \\
Agent 2 & 1.138 & 1.138 & 0.0 & 1.138 \\
Agent 3 & 1.049 & 1.070 & 2.0 & 1.069 \\
\hline
\end{tabular}

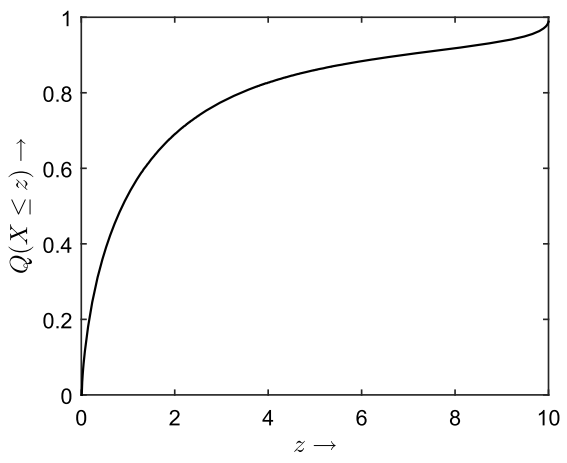

(a) Equilibrium price (algorithm)

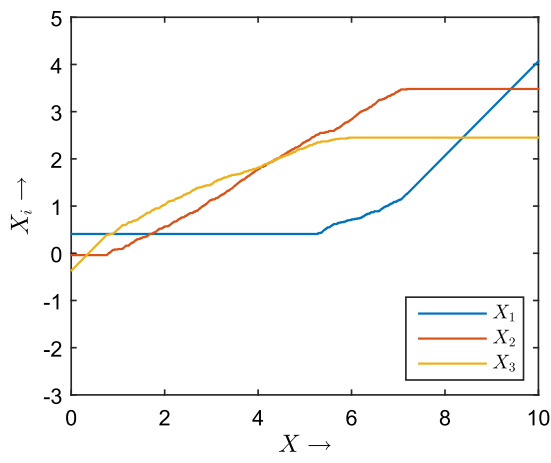

(b) Equilibrium allocation (algorithm)

Fig. 7 The equilibrium (CCE) solution of the algorithm corresponding to Example 4. The left figure displays the equilibrium price $\mathbb{Q}(X \leq z)$ (we display $\mathbb{Q}$ as $Q$ ), and the right figure displays the corresponding allocation

Example 4 (General rank-dependent utilities) Let $N=\{1,2,3\}$, and the three agents use an exponential utility function with parameters $\theta_{1}=3, \theta_{2}=2$ and $\theta_{3}=1$. Moreover, the agents use the distortion function $g_{i}(\cdot)=g\left(\cdot ; \gamma_{i}\right)$, with $\gamma_{1}=0.4, \gamma_{2}=$ 0.6 , and $\gamma_{3}=0.8$. So, Agent 1 (Agent 3) has the most (least) distorted probability measure, but is least (most) risk averse.

In Fig. 7, we show the outcomes of the algorithm of Sect. 5. We find again that equilibrium (CCE) price is inverse-S shaped. In particular, we find that including a strictly concave utility function yields higher equilibrium prices, as the price $\mathbb{Q}(X \leq z)$ is larger than this price in Example 1 for all $z$. Agent 1 (Agent 3) has the most (least) distorted probability measure. For that reason, we find in Fig. 7 that Agent 1 bears the least risk in the worst and best realizations of the total endowments $X$, and in turn, it has a low allocation in case the realizations of $X$ are around the average. Moreover, we find the opposite pattern for Agent 3. Moreover, the equilibrium (CCE) allocation for Agent 2 is S-shaped, and thus neither convex or concave. Such S-shaped allocation is commonly observed in an insurance market, which requires comonotonic contracts as we discussed in Sect. 1.1.

In Table 4, we show the certainty equivalents of the equilibrium (CCE) allocations. In particular, we find that the equilibrium is most attractive for the agent with the most distorted probability measure (Agent 1) and for the most risk averse agent (Agent 3). 
Table 4 The certainty equivalents before and after risk sharing, corresponding to Example 4

\begin{tabular}{lllc}
\hline & $\mathrm{CEQ}_{i}^{\text {prior }}$ & $\mathrm{CEQ}_{i}^{\text {post }}($ algorithm) & \% increase \\
\hline Agent 1 & 0.75 & 0.90 & 19.3 \\
Agent 2 & 1.10 & 1.14 & 3.0 \\
Agent 3 & 1.11 & 1.19 & 6.8 \\
\hline
\end{tabular}

\section{Concluding remarks}

In this paper, we introduce the novel concept of comonotone markets, and study competitive equilibria (CCE) in such markets for DU and RDU preferences. The comonotone market is closely associated with a complete market, in the sense that competitive equilibria in the complete market are necessarily (but not sufficiently) equilibria in the corresponding comonotone market. Although these two markets are closely related, many of our findings on the comonotone market are in sharp contrast to results on the complete market, in terms of existence, uniqueness, closed-form solutions of the equilibria, and comonotonicity of the pricing kernel. For instance, we show existence of equilibria in comonotone markets under mild conditions, whereas existence in complete markets is known to hold only under stronger assumptionsboth for DU and RDU preferences. With the help of the comonotone market, we enhance the understanding of real financial and insurance markets, which are much more complicated objects.

We also design an algorithm which produces competitive equilibria numerically in the comonotone market for DU and RDU agents, and its numerical performance is illustrated to be quite satisfactory. Due to great technical challenges, theoretical properties of the algorithm, such as convergence and termination conditions for general RDU markets, are still unclear. We leave these questions for future study.

Acknowledgements The authors thank an anonymous referee, the Associate Editor, Mario Ghossoub, and Jianming Xia for helpful discussions on an earlier version of the paper.

Open Access This article is licensed under a Creative Commons Attribution 4.0 International License, which permits use, sharing, adaptation, distribution and reproduction in any medium or format, as long as you give appropriate credit to the original author(s) and the source, provide a link to the Creative Commons licence, and indicate if changes were made. The images or other third party material in this article are included in the article's Creative Commons licence, unless indicated otherwise in a credit line to the material. If material is not included in the article's Creative Commons licence and your intended use is not permitted by statutory regulation or exceeds the permitted use, you will need to obtain permission directly from the copyright holder. To view a copy of this licence, visit http://creativecommons.org/licenses/by/4.0/.

\section{A Appendix}

\section{A.1 The RDU-power market}

We use the same notation in the RDU markets of Sect. 4. Furthermore, we assume that all agents use power (including logarithmic) utility functions, given by 


$$
u_{i}(t)=\left\{\begin{array}{ll}
\frac{t^{1-\eta}-1}{1-\eta}, & \eta>0, \eta \neq 1 \\
\ln (t), & \eta=1
\end{array} \quad \text { for all } t>0\right.
$$

and $u_{i}(t)=-\infty$ if $t \leq 0$, for $i \in N$. We assume that the parameter $\eta$ is the same for all agents. We shall refer to this market as the RDU-power-comonotone market. In this market, agents are assumed to hold non-negative wealth all the time, and thus the equilibrium risk sharing will be conducted among non-negative random variables. For simplicity, we assume there exists some $\varepsilon>0$ such that $\xi_{1}, \ldots, \xi_{n}>\varepsilon$.

The solution to Problem (12), which is without the comonotonicity constraint, is given by

$$
X_{i}=\left(u_{i}^{\prime}\right)^{-1}\left(\frac{\mathrm{d} \mathbb{Q}}{\mathrm{d} Q_{i}} \lambda_{i}\right)=\left(\frac{\mathrm{d} Q_{i}}{\mathrm{~d} \mathbb{Q}} \frac{1}{\lambda_{i}}\right)^{1 / \eta}, \quad \text { where } \lambda_{i}^{-1 / \eta}=\frac{\mathbb{E}^{\mathbb{Q}}\left[\xi_{i}\right]}{\mathbb{E}^{\mathbb{Q}}\left[\left(\frac{\mathrm{d} Q_{i}}{\mathrm{~d} \mathbb{Q}}\right)^{1 / \eta}\right]}
$$

By the market clearing constraint,

$$
X=\sum_{i=1}^{n} X_{i}=\sum_{i=1}^{n}\left(\lambda_{i}^{-1} \frac{\mathrm{d} Q_{i}}{\mathrm{~d} \mathbb{Q}}\right)^{1 / \eta}=\left(\frac{\mathrm{d} \mathbb{P}}{\mathrm{d} \mathbb{Q}}\right)^{1 / \eta} \sum_{i=1}^{n}\left(\lambda_{i}^{-1} \frac{\mathrm{d} Q_{i}}{\mathrm{~d} \mathbb{P}}\right)^{1 / \eta}
$$

and then

$$
\frac{\mathrm{d} \mathbb{Q}}{\mathrm{d} \mathbb{P}}=\left(\frac{1}{X} \sum_{i=1}^{n}\left(\lambda_{i}^{-1} \frac{\mathrm{d} Q_{i}}{\mathrm{~d} \mathbb{P}}\right)^{1 / \eta}\right)^{\eta}
$$

Therefore, $X=\left(\frac{\mathrm{d} Q_{j}}{\mathrm{~d} \mathbb{Q}}\right)^{1 / \eta} \sum_{i=1}^{n}\left(\lambda_{i}^{-1} \frac{\mathrm{d} Q_{i}}{\mathrm{~d} Q_{j}}\right)^{1 / \eta}$, and, for $j=1, \ldots, n$,

$$
X_{j}=\frac{\lambda_{j}^{-1 / \eta} X}{\sum_{i=1}^{n}\left(\lambda_{i}^{-1} \frac{\mathrm{d} Q_{i}}{\mathrm{~d} Q_{j}}\right)^{1 / \eta}} .
$$

The comonotonicity constraint requires $X_{j} \in C(X)$ for all $j=1, \ldots, n$. Therefore, a sufficient condition for the existence of solution to the Problem (2) is

$$
\frac{X}{\sum_{i=1}^{n}\left(\lambda_{i}^{-1} \frac{\mathrm{d} Q_{i}}{\mathrm{~d} Q_{j}}\right)^{1 / \eta}} \text { is comonotonic with } X \text { for all } j=1, \ldots, n \text {. }
$$

Let $\bar{q}(x ; \eta)=\sum_{i=1}^{n}\left(\lambda_{i}^{-1} q_{i}(x)\right)^{1 / \eta}$ for $x>0$. The condition $\frac{\mathrm{d}}{\mathrm{d} x}\left(\frac{x q_{j}^{1 / \eta}(x)}{\bar{q}(x ; \eta)}\right) \geq 0$ or, equivalently,

$$
\sup _{x>0}\left\{x\left(\frac{\bar{q}^{\prime}(x ; \eta)}{\bar{q}(x ; \eta)}-\frac{1}{\eta} \inf _{j=1, \ldots, n}\left\{\frac{q_{j}^{\prime}(x)}{q_{j}(x)}\right\}\right)\right\} \leq 1
$$


guarantee that $\left(X_{1}^{*}, \ldots, X_{n}^{*}\right)$, given by (30) is a solution to the Problem (2).

This result is summarized in the following theorem.

Theorem 6 In the RDU-power-comonotone market, if (31) holds, then a CCE is uniquely given by $\left(\left(X_{1}, \ldots, X_{n}\right), \mathbb{Q}\right)$ in $(29)-(30)$.

\section{A.2 Proofs of theorems, lemmas and propositions}

Proof of Proposition 1 We write $Y_{i}=f_{i}(X)$ for $f_{i} \in \mathcal{F}$ (Denneberg 1994), and let $f_{i}^{-1}(y)=\inf \left\{t \in \mathbb{R}: f_{i}(t)>y\right\} \in \mathcal{F}$ be the inverse function of $f_{i}$. Then, $V_{i}\left(Y_{i}\right)=f_{i}^{-1}(0)+\int_{0}^{\infty} g_{i}\left(S_{X}(t)\right) f_{i}^{\prime}(t) \mathrm{d} t+\int_{-\infty}^{0}\left(g_{i}\left(S_{X}(z)\right)-1\right) f_{i}^{\prime}(t) \mathrm{d} t$ and $\mathbb{E}^{\mathbb{Q}}\left[Y_{i}\right]=f_{i}^{-1}(0)+\int_{0}^{\infty} \mathbb{Q}(X>t) f_{i}^{\prime}(t) \mathrm{d} t+\int_{-\infty}^{0}(\mathbb{Q}(X>t)-1) f_{i}^{\prime}(t) \mathrm{d} t$. Thus, for $Y_{i}=f_{i}(X) \in C(X), V_{i}\left(Y_{i}\right)-\mathbb{E}^{\mathbb{Q}}\left[Y_{i}\right]=\int_{-\infty}^{\infty}\left(g_{i}\left(S_{X}(t)\right)-\mathbb{Q}(X>t)\right) f_{i}^{\prime}(t) \mathrm{d} t$. Since $R(X)$ is a closed interval, maximizing this over the set $\mathcal{F}$ yields the desired result.

Proof of Theorem 1 “if” part. Consider functions $f_{i}^{\prime}, i \in N$ satisfying (5). For each $z \in R(X)$, if $i=\min \left\{l \in N: g_{l}\left(S_{X}(z)\right) \geq \mathbb{Q}(X>z)\right\}$, then take $f_{i}^{\prime}(z)=1$ and $f_{j}^{\prime}(z)=0$ for $j \neq i$. It follows that $\sum_{i=1}^{n} f_{i}^{\prime}(z)=1$. Write $X_{i}^{*}=f_{i}(X)-$ $\mathbb{E}^{\mathbb{Q}}\left[f_{i}(X)\right]+\mathbb{E}^{\mathbb{Q}}\left[\xi_{i}\right]$ almost surely where $f_{i}(z)=\int_{0}^{z} f_{i}^{\prime}(t) \mathrm{d} t$. Here, we take $f_{i}(0)=0$ without loss of generality. It is easy to check that $\sum_{i=1}^{n} f_{i}(z)=z$ for $z \in R(X)$. By Lemma 1 and Proposition $1, X_{i}^{*}$ is the maximizer of (2) for each $i \in N$. Meanwhile, $\sum_{i=1}^{n} X_{i}^{*}=\sum_{i=1}^{n} f_{i}(X)-\sum_{i=1}^{n} \mathbb{E}^{\mathbb{Q}}\left[f_{i}(X)\right]+\sum_{i=1}^{n} \mathbb{E}^{\mathbb{Q}}\left[\xi_{i}\right]=X$. Therefore, the pair $\left(\left(X_{1}^{*}, \ldots, X_{n}^{*}\right), \mathbb{Q}\right)$ is a competitive equilibrium.

"only if" part. Write $X_{i}^{*}=f_{i}(X)$ where $f_{i} \in \mathcal{F}, i \in N$. Again this is possible by Denneberg's Lemma. Since $\left(\left(X_{1}^{*}, \ldots, X_{n}^{*}\right), \mathbb{Q}\right)$ is a competitive equilibrium, we have $\sum_{i=1}^{n} f_{i}^{\prime}(t)=1$ for almost everywhere $t \in R(X)$.

(i) Suppose that $\mathbb{Q}(X>z)>g_{N, 1}\left(S_{X}(z)\right)$ for some $z \in R(X)$. Since both $\mathbb{Q}(X>$ .) and $g\left(S_{X}(\cdot)\right)$ are decreasing functions, and $\mathbb{Q}(X>\cdot)$ is right-continuous, there exists a closed interval $[z, z+\varepsilon] \subset R(X)$ on which $\mathbb{Q}(X>t)>g_{N, 1}\left(S_{X}(t)\right)$. Applying Proposition 1 , we get $f_{i}^{\prime}(z)=0$ for each $i \in N$ on $[z, z+\varepsilon]$. Noting that $[z, z+\varepsilon]$ has non-zero measure, this contradicts $\sum_{i=1}^{n} f_{i}^{\prime}(t)=1$ for almost everywhere $t \in R(X)$.

(ii) Suppose that $\mathbb{Q}(X>z)<g_{i}\left(S_{X}(z)\right)$ for some $z \in R(X)$ and $i \in M$ and $M$ has a cardinality strictly greater than 1 . Since both $\mathbb{Q}(X>\cdot)$ and $g_{i}\left(S_{X}(\cdot)\right)$ are decreasing and right-continuous functions, there exists a closed interval $[z, z+$ $\varepsilon] \subset R(X)$ on which $\mathbb{Q}(X>z)<g_{i}\left(S_{X}(z)\right)$ for $i \in M$. By Proposition 1 , we get $f_{i}^{\prime}(z)=1$ for $i \in M$ on $[z, z+\varepsilon]$. Noting that $[z, z+\varepsilon]$ has non-zero measure, and each $f_{i}^{\prime}$ is non-negative on the convex range $R(X)$, this contradicts $\sum_{i=1}^{n} f_{i}^{\prime}(t)=1$ for almost everywhere $t \in R(X)$.

Combining (i) and (ii) completes the proof.

Proof of Theorem 2 (i) Noting that $g_{N, 2}\left(S_{X}(z)\right) \leq g_{N, 1}\left(S_{X}(z)\right)$, there always exists $\mathbb{Q}$ satisfying $g_{N, 2}\left(S_{X}(z)\right) \leq \mathbb{Q}(X>z) \leq g_{N, 1}\left(S_{X}(z)\right)$. For such $\mathbb{Q}$, it is easy to see that $f_{1}, \ldots, f_{n}$ satisfying $(5)$ with $\sum_{i=1}^{n} f_{i}(X)=X$ also exist. By Theorem 1, a CCE exists. 
(ii) Assume $g_{N, 1}(t)>g_{N, 2}(t)$ for a.e. $t \in[0,1]$. In this case, by Theorem 1 (i), $\mathbb{Q}$ can be anything such that $g_{N, 2}\left(S_{X}(z)\right) \leq \mathbb{Q}(X>z) \leq g_{N, 1}\left(S_{X}(z)\right)$, which is not unique. Moreover, for $\mathbb{Q}$ satisfying the above condition, (5) and the condition $\sum_{i=1}^{n} f_{i}(X)=X$ uniquely determine the functions $f_{1}, \ldots, f_{n}$. Therefore, the equilibrium allocation is unique up to the constant term $-\mathbb{E}^{\mathbb{Q}}\left[f_{i}(X)\right]+\mathbb{E}^{\mathbb{Q}}\left[\xi_{i}\right]$ as specified in Theorem 1 (ii).

(iii) Suppose that $g_{N, 1}(t)=g_{N, 2}(t)$ for almost everywhere $t \in[0,1]$. Since $\mathbb{Q}(X>z)$ is a right-continuous function of $z$, it is uniquely determined by $g_{N, 2}\left(S_{X}(z)\right) \leq \mathbb{Q}(X>z) \leq g_{N, 1}\left(S_{X}(z)\right)$. As $\mathcal{B}$ is the set of all events generated by $X, \mathbb{Q}$ is uniquely determined by $\mathbb{Q}(X>z), z \in R(X)$. In this case, $f_{1}, \ldots, f_{n}$ satisfying (5) and $\sum_{i=1}^{n} f_{i}(X)=X$ are not unique due to the fact that for $i, j \in N, i \neq j$ such that $g_{i}=g_{N, 1}=g_{N, 2}=g_{j}, f_{i}^{\prime}$ and $f_{j}^{\prime}$ in (5) can be arbitrary as long as they add up to 1 .

Proof of Theorem 3 (i) It follows from the same proof of Theorem 4 (ii) below.

(ii) Suppose that $\left(X_{1}, \ldots, X_{n}\right) \in \mathbb{A}_{n}^{c}(X)$ is a Pareto-optimal allocation. Write $X_{i}=$ $f_{i}(X)$ where each $f_{i}$ is in $\mathcal{F}, i \in N$. Take $\mathbb{Q}$ such that $\mathbb{Q}(X>z)=g_{N, 1}\left(S_{X}(z)\right)$ for $z \in R(X)$, where $g_{N, 1}(t)=\max \left\{g_{i}(t): i \in N\right\}$ for $t \in[0,1]$. By Theorem 1 , it suffices to show that $f_{i}^{\prime}$ satisfies (5), since we already have $\sum_{i=1}^{n} f_{i}^{\prime}(z)=1$ for $z \in R(X)$ by the definition of an allocation $\left(X_{1}, \ldots, X_{n}\right)$. By direct calculation, we have

$$
\sum_{i=1}^{n} V_{i}\left(X_{i}\right)=\sum_{i=1}^{n} \int_{0}^{\infty} g_{i}\left(S_{X}(t)\right) f_{i}^{\prime}(t) \mathrm{d} t .
$$

By Proposition 2 and (7), we have, since $\left(X_{1}, \ldots, X_{n}\right)$ is Pareto-optimal,

$$
\sum_{i=1}^{n} V_{i}\left(X_{i}\right)=D_{g_{N, 1}}(X)=\int_{0}^{\infty} g_{N, 1}\left(S_{X}(t)\right) \mathrm{d} t
$$

Noting that $\sum_{i=1}^{n} f_{i}^{\prime}(z)=1$ almost everywhere for $z \in R(X)$, and combining (32) and (33), we have $f_{i}^{\prime}(z)=0$ almost everywhere for $g_{i}\left(S_{X}(z)\right)<$ $\mathbb{Q}(X>z)$. Further note that by the definition of $\mathbb{Q}$ we always have $g_{i}\left(S_{X}(z)\right) \leq$ $\mathbb{Q}(X>z)$. Therefore, $f_{i}^{\prime}(z)$ satisfies (5).

Proof of Proposition 3 Denote $M_{i}=\sup _{Y \in C(X)} V_{i}\left(Y-\mathbb{E}^{\mathbb{Q}}[Y]+\mathbb{E}^{\mathbb{Q}}\left[\xi_{i}\right]\right)$, and take a sequence $Y_{i, k} \in C(X), k \in \mathbb{Z}^{+}$such that $\lim _{k \rightarrow \infty} V_{i}\left(Y_{i, k}-\mathbb{E}^{\mathbb{Q}}\left[Y_{i, k}\right]+\mathbb{E}^{\mathbb{Q}}\left[\xi_{i}\right]\right)=M_{i}$. Write $Y_{i, k}=f_{i, k}(X), k \in \mathbb{Z}^{+}$where $f_{i, k} \in \mathcal{F}$. Without loss of generality, assume $f_{i, k}(0)=0, k \in \mathbb{Z}^{+}$. Using Lemma 2.3 of Cai et al. (2017), there exists a subsequence of $\left\{f_{i, k}\right\}$ converges point-wise to some $f_{i}^{*} \in \mathcal{F}$. For notational simplicity, write $f_{i, k} \rightarrow f_{i}^{*}$ point-wise, and then $f_{i, k}(X) \rightarrow f_{i}^{*}(X)$ almost surely. Consequently, $\lim _{k \rightarrow \infty} \mathbb{E}^{\mathbb{Q}}\left[f_{k}(X)\right]=\mathbb{E}^{\mathbb{Q}}\left[f^{*}(X)\right]$. Denote $c_{i, k}=\mathbb{E}^{\mathbb{Q}}\left[\xi_{i}\right]-\mathbb{E}^{\mathbb{Q}}\left[f_{i, k}(X)\right]$ and $c_{i}^{*}=$ $\mathbb{E}^{\mathbb{Q}}\left[\xi_{i}\right]-\mathbb{E}^{\mathbb{Q}}\left[f_{i}^{*}(X)\right]$. By Fatou's Lemma, for $i \in N$,

$$
M_{i}=\lim _{k \rightarrow \infty} V_{i}\left(Y_{i, k}+c_{i, k}\right) \leq \limsup _{k \rightarrow \infty} V_{i}\left(f_{i, k}(X)+c_{i, k}\right) \leq V_{i}\left(f_{i}^{*}(X)+c_{i}^{*}\right) \leq M_{i},
$$


which completes the proof.

Proof of Lemma 2 First note that we can write $\xi_{i}=\varphi_{i}(X)$ for a bounded and continuous function $\varphi_{i}$ defined on $R(X), i=1, \ldots, n$. Moreover, we have $\mathbb{E}^{Q^{k}}\left[\xi_{i, k}\right]=$ $\mathbb{E}^{k}\left[X_{i, k}^{*}\right]$ for $i \in N$ and we can write $X_{i, k}^{*}=f_{i, k}\left(X^{k}\right)$ for some functions $f_{i, k} \in \mathcal{F}$, $i \in N$, where $X^{k}=\xi_{1, k}+\cdots+\xi_{n, k}$. It is clear that $\left\{X^{k}\right\}_{k=1}^{\infty}$ converges to $X$ uniformly.

Let $\mathcal{B}(R(X))$ be the Borel $\sigma$-algebra of $R(X)$. Each measure $Q^{k}$ on $\mathcal{B}$ induces a measure $\tilde{Q}^{k}$ on $\mathcal{B}(R(X))$ such that $\tilde{Q}^{k}(A)=Q^{k}\left(X^{-1}(A)\right)$ for all subset $A \in$ $\mathcal{B}(R(X))$. Since $R(X)$ is a compact subset of $\mathbb{R}$, every collection of measures on $\mathcal{B}(R(X))$ is tight. Prokhorov's theorem implies that there exists a subsequence of $\left\{\tilde{Q}^{k}\right\}_{k=1}^{\infty}$ such that

$$
\tilde{Q}^{k_{l}} \rightarrow \tilde{Q}^{*} \text { weakly as } l \rightarrow \infty \text { for some measure } \tilde{Q}^{*} \text { on } \mathcal{B}(R(X)) .
$$

For notational simplicity, we choose this subsequence as $\left\{\left(\left(X_{1, k}^{*}, \ldots, X_{n, k}^{*}\right), Q^{k}\right)\right\}_{k=1}^{\infty}$ in the following. Define $\mathbb{Q}^{*}(B)=\tilde{Q}^{*}(X(B))$ for $B \in \mathcal{B}$. Then, $\mathbb{E} Q^{k}\left[\xi_{i}\right]=$ $\int \varphi_{i}(x) \tilde{Q}^{k}(\mathrm{~d} x)$ converges to $\mathbb{E}^{\mathbb{Q}^{*}}\left[\xi_{i}\right]=\int \varphi_{i}(x) \tilde{Q}^{*}(\mathrm{~d} x)$ since the function $\varphi_{i}$ is bounded and continuous. Moreover, since $\left\{\xi_{i, k}\right\}_{k=1}^{\infty}$ converges to $\xi_{i}$ uniformly, then for any $\varepsilon>0$, there exists $K \in \mathbb{N}$ such that $\left|\mathbb{E}^{Q^{k}}\left[\xi_{i, k}\right]-\mathbb{E}^{Q^{k}}\left[\xi_{i}\right]\right| \leq \varepsilon$ for all $k>K$. Therefore, we get $\lim _{k \rightarrow \infty} \mathbb{E}^{Q^{k}}\left[\xi_{i, k}\right]=\mathbb{E}^{\mathbb{Q}^{*}}\left[\xi_{i}\right]$.

Note that $\tilde{f}_{1, k}=f_{1, k}-f_{1, k}(0)$ are functions in $\mathcal{F}$ with $\tilde{f}_{1, k}(0)=0$, using Lemma 2.3 of Cai et al. (2017), it has a subsequence $\tilde{f}_{1, k}$ which converges point-wise to some $\tilde{f}_{1}^{*} \in \mathcal{F}$ and $\tilde{f}_{1}^{*}(0)=0$. Doing this sequentially for $i \in N$, we get a subsequence such that $\left\{\tilde{f}_{i, k_{l}}\right\}_{l=1}^{\infty}$ point-wise converges to a function $\tilde{f}_{i}^{*}$ for all $i \in N$ simultaneously. As $\tilde{f}_{i}^{*}$ is uniformly bounded and continuous, $\tilde{f}_{i}^{*}\left(X^{k}\right)$ converges to $\tilde{f}_{i}^{*}(X)$ uniformly. Combining the above results, $\tilde{f}_{i, k_{l}}\left(X^{k}\right)$ converges to $\tilde{f}_{i}^{*}(X)$ uniformly for all $i \in$ $N$ simultaneously. Recall from (34) and the explanation afterward that $\left\{\tilde{Q}^{k}\right\}_{k \in \mathbb{N}}$ is chosen such that $\tilde{Q}^{k} \rightarrow \tilde{Q}^{*}$ weakly. Therefore, we obtain the joint convergence that $\tilde{f}_{i, k_{l}}\left(X^{k}\right)$ converges to $\tilde{f}_{i}^{*}(X)$ uniformly and $\tilde{Q}^{k_{l}} \rightarrow \tilde{Q}^{*}$ weakly as $l \rightarrow \infty$. Again, for notational simplicity, we choose this subsequence as $\left\{\left(\left(X_{1, k}^{*}, \ldots, X_{n, k}^{*}\right), Q^{k}\right)\right\}_{k=1}^{\infty}$ in the following. Similarly to the case of $\xi_{i}$, one can show $\lim _{k \rightarrow \infty} \mathbb{E}^{Q^{k}}\left[\tilde{f}_{i, k}(X)\right]=$ $\mathbb{E}^{\mathbb{Q}^{*}}\left[\tilde{f}_{i}^{*}(X)\right]$. Using $f_{i, k}(0)=\mathbb{E}^{Q^{k}}\left[\xi_{i, k}\right]-\mathbb{E}^{Q^{k}}\left[\tilde{f}_{i, k}(X)\right]$, we have $\lim _{k \rightarrow \infty} f_{i, k} \rightarrow$ $f_{i}^{*}=\tilde{f}_{i}^{*}+f_{i}^{*}(0)$ point-wise, where $f_{i}^{*}(0)=\mathbb{E}^{\mathbb{Q}^{*}}\left[\xi_{i}\right]-\mathbb{E}^{\mathbb{Q}^{*}}\left[\tilde{f}_{i}^{*}(X)\right]$. Let $X_{i}^{*}=$ $f_{i}^{*}(X)$ for $i \in N$. Then $X_{i, k}$ converges to $X_{i}^{*}$ uniformly and $\mathbb{E}^{\mathbb{Q}^{*}}\left[X_{i}^{*}\right]=\mathbb{E}^{\mathbb{Q}^{*}}\left[\xi_{i}\right]$, i.e., the budget constraint for each agent holds for $\left(X_{i}^{*}, \ldots, X_{n}^{*}\right)$, which can be easily verified to be an allocation in $\mathbb{A}^{c}(X)$.

To show that $\left(\left(X_{1}^{*}, \ldots, X_{n}^{*}\right), \mathbb{Q}^{*}\right)$ is indeed a competitive equilibrium, it remains to show that for any $Y_{i} \in C(X) \cap B\left(\mathbb{Q}^{*}, \xi_{i}\right)$, it holds that $V_{i}\left(Y_{i}\right) \leq V_{i}\left(X_{i}^{*}\right), i \in N$. To this end, we first fix $i \in N$ and take an arbitrary $Z_{i} \in C(X) \cap B\left(\mathbb{Q}^{*}, \xi\right)$ such that $\mathbb{E}^{\mathbb{Q}^{*}}\left[Z_{i}\right]<\mathbb{E}^{\mathbb{Q}^{*}}\left[\xi_{i}\right]$.

Write $Z_{i}=z_{i}(X)$ for some function $z_{i} \in \mathcal{F}$ and let $Z_{i, k}=z_{i}\left(X_{k}\right), k \in \mathbb{N}$. Take $\varepsilon=\left(\mathbb{E}^{\mathbb{Q}^{*}}\left[\xi_{i}\right]-\mathbb{E}^{\mathbb{Q}^{*}}\left[Z_{i}\right]\right) / 3$. Using the convergence results analyzed above, there exist $K_{1}, K_{2}$ and $K_{3}$ such that $\left|\mathbb{E}^{Q^{k}}\left[Z_{i, k}\right]-\mathbb{E}^{\mathbb{Q}^{*}}\left[Z_{i, k}\right]\right| \leq \varepsilon$ for all $k>K_{1}$, $\left|\mathbb{E}^{\mathbb{Q}^{*}}\left[Z_{i, k}\right]-\mathbb{E}^{\mathbb{Q}^{*}}\left[Z_{i}\right]\right| \leq \varepsilon$ for all $k>K_{2}$, and $\left|\mathbb{E}^{\mathbb{Q}^{*}}\left[\xi_{i}\right]-\mathbb{E}^{Q^{k}}\left[\xi_{i, k}\right]\right| \leq \varepsilon$ for all 
$k>K_{3}$. Taking $K=\max \left\{K_{1}, K_{2}, K_{3}\right\}$, we have, for all $k>K$,

$$
\mathbb{E}^{Q^{k}}\left[Z_{i, k}\right] \leq \mathbb{E}^{\mathbb{Q}^{*}}\left[Z_{i, k}\right]+\varepsilon \leq \mathbb{E}^{\mathbb{Q}^{*}}\left[Z_{i}\right]+2 \varepsilon=\mathbb{E}^{\mathbb{Q}^{*}}\left[\xi_{i}\right]-\varepsilon \leq \mathbb{E}^{Q^{k}}\left[\xi_{i, k}\right]
$$

Therefore, from the optimality of $X_{i, k}$ as it is in an equilibrium, we have $V_{i}\left(Z_{i, k}\right) \leq$ $V_{i}\left(X_{i, k}\right)$. Note that $X \leq X^{k}$ implies $Z_{i} \leq Z_{i, k}$, and we have $\lim _{\sup _{k \rightarrow \infty}} V_{i}\left(X_{i, k}\right) \leq$ $V_{i}\left(X_{i}^{*}\right)$ by Fatou's lemma, as $u_{i}\left(X_{i}\right)$ is bounded above and $X_{i, k} \rightarrow X_{i}^{*}$ uniformly as $k \rightarrow \infty$. It follows that

$$
V_{i}\left(Z_{i}\right) \leq \limsup _{k \rightarrow \infty} V_{i}\left(Z_{i, k}\right) \leq \limsup _{k \rightarrow \infty} V_{i}\left(X_{i, k}\right) \leq V_{i}\left(X_{i}^{*}\right)
$$

Thus, for all $Z_{i} \in C(X)$ such that $\mathbb{E}^{\mathbb{Q}^{*}}\left[Z_{i}\right]<\mathbb{E}^{\mathbb{Q}^{*}}\left[\xi_{i}\right]$, we have $V_{i}\left(Z_{i}\right) \leq V_{i}\left(X_{i}^{*}\right)$.

Next, take $Y_{i} \in C(X) \cap B\left(\mathbb{Q}^{*}, \xi_{i}\right)$. Let $W_{i, m}=\max \left\{Y_{i}, m\right\}$ for $m \in \mathbb{R}$ and $r_{i}=\sup \left\{y \in \mathbb{R}: \mathbb{P}\left(Y_{i}>y\right)>0\right\}$. If $V_{i}\left(Y_{i}\right)=-\infty$, then there is nothing to show. If $r_{i}>d_{i}$, then $\mathbb{E}^{\mathbb{Q}^{*}}\left[W_{i, m}\right]<\mathbb{E}^{\mathbb{Q}^{*}}\left[\xi_{i}\right]$ for $d_{i}<m<r_{i}$, and hence $V_{i}\left(W_{i, m}\right) \leq V_{i}\left(X_{i}^{*}\right)$. Therefore, $V_{i}\left(Y_{i}\right)=\lim _{m \uparrow r_{i}} V_{i}\left(W_{i, m}\right) \leq V_{i}\left(X_{i}^{*}\right)$ by the continuity of $u_{i}$ on $\left(d_{i}, \infty\right)$. If $r_{i}=d_{i}$ and $V_{i}\left(Y_{i}\right)>-\infty$, then $Y_{i}=d_{i}$ almost surely and $V_{i}\left(d_{i}\right)>-\infty$. Since $\xi_{i, k} \geq d_{i}$, we have $V_{i}\left(X_{i, k}\right) \geq V_{i}\left(d_{i}\right)>-\infty$, implying $X_{i, k} \geq d_{i}$ almost surely. Therefore, $X_{i}^{*} \geq d_{i}$ almost surely, and $V_{i}\left(X_{i}^{*}\right) \geq V_{i}\left(d_{i}\right)=V_{i}\left(Y_{i}\right)$. Combining all cases, we have $V_{i}\left(Y_{i}\right) \leq V_{i}\left(X_{i}^{*}\right)$, and hence $\left(\left(X_{1}^{*}, \ldots, X_{n}^{*}\right), \mathbb{Q}^{*}\right)$ is a competitive equilibrium.

Proof of Theorem 4 We will switch between discrete and continuous models in the proof, and these models are connected by Lemma 2.

(i) First, suppose that the probability space $(\Omega, \mathcal{B}, \mathbb{P})$ is such that $\Omega$ is finite. Note that $C(X)$ is non-empty, convex, and bounded, and the preference induced by $R_{u_{i}, g_{i}}$ is continuous and concave on the admissible set $C(X)$. The constant $Y=1$ is a useful commodity bundle (see Werner 1987 for a definition), because $R_{u_{i}, g_{i}}$ is monotone. Also, for every $\mathbb{Q} \in \mathcal{P}$ and $i \in N$, it holds $\mathbb{E}^{\mathbb{Q}}\left[\zeta_{i}\right]>$ $\inf _{Y \in C(X)} \mathbb{E}^{\mathbb{Q}}[Y]$ because $\zeta_{i}$ is assumed to be bounded, and $Y=f(X) \leq X+$ $f(0)$ implies for $f(0) \rightarrow-\infty$ that $\inf _{Y \in C(X)} \mathbb{E}^{\mathbb{Q}}[Y]=-\infty$. Moreover, there exists a pricing measure that admits no arbitrage opportunities for all agents; namely take a pricing measure that is equivalent to $\mathbb{P}$. Then, existence of a competitive equilibrium follows from Theorem 1 of Werner (1987).

Next, we show existence for an infinite $\Omega$. To this end, we first construct a sequence of $\left\{X^{k}\right\}_{k \in \mathbb{N}}$ uniformly converging to $X$ and $X^{k}, k \in \mathbb{N}$, takes finitely many values. Again, the existence of equilibrium associated with $X_{k}$ follows from Theorem 1 of Werner (1987). Then, Lemma 2 guarantees the limit of equilibria associated with $X_{k}$ is an equilibrium for the original model. For $k \in \mathbb{N}$, consider a partition $\left\{I_{k, 1}, \ldots, I_{k, k}\right\}$ of $R(X)$, that is $R(X)=\cup_{m=1}^{k} I_{k, m}$ and $I_{k, m} \cap I_{k, l}=\emptyset$ for $m \neq l$, such that each $I_{k, m}$ is an interval with the Lebesgue measure smaller than $\frac{\|R(X)\|}{k-1}$, where $\|R(X)\|$ is the length of $R(X)$. Write $a_{k, m}=\sup I_{k, m}$ and $A_{k, m}=X^{-1}\left(I_{k, m}\right)$ for $m=1, \ldots, k$. For each $i \in N$, write $\xi_{i}=\varphi_{i}(X)$, where $\varphi_{i}: R(X) \rightarrow \mathbb{R}$ is a bounded and continuous function on a compact interval, 
making it uniformly continuous. Define

$$
\xi_{i, k}=\sum_{m=1}^{k} \varphi_{i}\left(a_{k, m}\right) \mathbb{I}_{\left\{X \in I_{k, m}\right\}}=\sum_{m=1}^{k} \varphi_{i}\left(a_{k, m}\right) \mathbb{I}_{A_{k, m}}, \quad i \in N, k \in \mathbb{N} .
$$

Since $\varphi_{i}$ is uniformly continuous, it is easy to see that $\left\{\xi_{i, k}\right\}_{k=1}^{\infty}$ converges to $\xi_{i}$ uniformly for $i \in N$. For each $k \in \mathbb{N}$, define $X^{k}=\sum_{i \in N} \xi_{i, k}$. Note that $X^{k} \geq X$ for $k \in \mathbb{N}$ by construction. By using the existence result of Werner (1987) mentioned above, there exists an equilibrium $\left(\left(X_{1, k}^{*}, \ldots, X_{n, k}^{*}\right), Q^{k}\right)$ for the initial endowments $\left(\xi_{1, k}, \ldots, \xi_{n, k}\right)$ in the probability space generated by $\sigma\left(X^{k}\right)$. By Lemma 2 we obtain the existence of a competitive equilibrium for the original model. ${ }^{13}$

(ii) This is a classic market where the non-satiation condition holds, and hence Pareto efficiency of competitive equilibria should be expected. For completeness we provide a self-contained proof. Let $\left(\left(X_{1}, \ldots, X_{n}\right), \mathbb{Q}\right) \in \mathbb{A}_{n}^{c}(X) \times \mathcal{P}$ be a CCE for this market. Hence

$$
X_{i} \in \underset{Y \in C(X) \cap B\left(\mathbb{Q}, \xi_{i}\right)}{\arg \max } V_{i}(Y), \quad i \in N .
$$

Suppose, for the purpose of contradiction, that $\left(X_{1}, \ldots, X_{n}\right)$ is not Paretooptimal. Then, by definition of Pareto optimality, there exists an allocation $\left(X_{1}^{\prime}, \ldots, X_{n}^{\prime}\right) \in \mathbb{A}_{n}^{c}(X)$ such that $V_{i}\left(X_{i}^{\prime}\right) \geq V_{i}\left(X_{i}\right)$ for all $i \in N$ and $V_{j}\left(X_{j}^{\prime}\right)>V_{j}\left(X_{j}\right)$ for some $j \in N$. If $\mathbb{E}^{\mathbb{Q}}\left[X_{j}^{\prime}\right] \leq \mathbb{E}^{\mathbb{Q}}\left[X_{j}\right]$, then (35) does not hold for $i=j$, thus a contradiction to the definition of $\left(\left(X_{1}, \ldots, X_{n}\right), \mathbb{Q}\right)$ being a competitive equilibrium. Therefore, $\mathbb{E}^{\mathbb{Q}}\left[X_{j}^{\prime}\right]>\mathbb{E}^{\mathbb{Q}}\left[X_{j}\right]$. Then, since $\sum_{i=1}^{n} \mathbb{E}^{\mathbb{Q}}\left[X_{i}^{\prime}\right]=\mathbb{E}^{\mathbb{Q}}[X]=\sum_{i=1}^{n} \mathbb{E}^{\mathbb{Q}}\left[X_{i}\right]$, there exists some $k \in N$ such that $\mathbb{E}^{\mathbb{Q}}\left[X_{k}^{\prime}\right]<\mathbb{E}^{\mathbb{Q}}\left[X_{k}\right]$. Now, let $X_{k}^{*}=X_{k}^{\prime}+\mathbb{E}^{\mathbb{Q}}\left[X_{k}\right]-\mathbb{E}^{\mathbb{Q}}\left[X_{k}^{\prime}\right]$, which clearly satisfies $\mathbb{E}^{\mathbb{Q}}\left[X_{k}^{*}\right]=\mathbb{E}^{\mathbb{Q}}\left[X_{k}\right]$, and thus $X_{k}^{*} \in B\left(\mathbb{Q}, \xi_{k}\right)$. From the definition of $V_{k}$ where $u_{k}$ is strictly increasing and $V_{k}\left(X_{k}\right)>-\infty$, we have $V_{k}\left(X_{k}^{*}\right)>V_{k}\left(X_{k}^{\prime}\right) \geq V_{k}\left(X_{k}\right)$. As a consequence, (35) does not hold for $i=k$, thus a contradiction to the definition of $\left(\left(X_{1}, \ldots, X_{n}\right), \mathbb{Q}\right)$ being a CCE. In summary, $\left(X_{1}, \ldots, X_{n}\right)$ is Pareto-optimal.

(iii) Suppose that $\left(X_{1}, \ldots, X_{n}\right)$ is a Pareto-optimal allocation. Since the nonsatiation condition holds, it is clear that $\left(X_{1}, \ldots, X_{n}\right)$ is proper. As the set of allocations $\mathbb{A}^{c}(X)$ is convex, there exist $\lambda_{1}, \ldots, \lambda_{n}>0$ such that $\left(X_{1}, \ldots, X_{n}\right)$ maximizes $\sum_{i=1}^{n} \lambda_{i} V_{i}\left(Y_{i}\right)$ over $\left(Y_{1}, \ldots, Y_{n}\right) \in \mathbb{A}^{c}(X)$. Write $V\left(Y_{1}, \ldots, Y_{n}\right)=\sum_{i=1}^{n} \lambda_{i} V_{i}\left(Y_{i}\right)$ for $\left(Y_{1}, \ldots, Y_{n}\right) \in \mathbb{A}^{c}(X)$. Note that $V$ is a strictly concave function on $\mathbb{A}^{c}(X)$. We define $X^{k}$ as in part (i) and note that $X^{k} \geq X$. Let $\left(X_{1, k}, \ldots, X_{n, k}\right)$ be a maximizer of $V$ over $\mathbb{A}^{c}\left(X^{k}\right)$, and hence it is Pareto-optimal for the comonotone market with total risk $X^{k}$. Since

13 The set of natural numbers with the ordinary order is a directed set, so that $\left(\left(X_{1, k}^{*}, \ldots, X_{n, k}^{*}\right), Q^{k}\right)$ form a net. Lemma 2 shows that this net contains a subnet whose components converge. In Bewley (1972), the author also used the net-convergence argument to show the existence of an equilibrium for economies whose commodity space has infinite dimension. 
$V$ is strictly concave and continuous, and due to our construction of $X^{k}$, we can see that $\left(X_{1, k}, \ldots, X_{n, k}\right) \rightarrow\left(X_{1}, \ldots, X_{n}\right)$ uniformly. By Propositions 16.D.1 and 16.D.3 of Mas-Colell et al. (1995), the Pareto-optimal allocation $\left(X_{1, k}, \ldots, X_{n, k}\right)$ is also an equilibrium allocation. By Lemma 2, a subsequence of $\left(X_{1, k}, \ldots, X_{n, k}\right)$ converges to an equilibrium allocation, which shows that $\left(X_{1}, \ldots, X_{n}\right)$ is an equilibrium allocation.

\section{A.3 Convergence of the algorithm in a DU-comonotone market}

We use the following notation for the convergence result of the algorithm in a DUcomonotone market. In a DU-comonotone market, for each positive integer $m$, let $\left(\left(\psi_{m}^{1}(\hat{X}), \ldots, \psi_{m}^{n}(\hat{X})\right), \hat{Q}_{m}\right)$ be the output of the algorithm, where $\psi_{m}^{i}(\hat{X})$ for $i \in N$ and $\hat{Q}_{m}$ are given in the step (3). Moreover, as $m \rightarrow \infty$, denote by $f_{i}(x)$ the pointwise limit of $\psi_{m}^{i}(x)$ for $i \in N$, and denote by $Q(X>x)$ the point-wise limit of $\hat{Q}_{m}\left(\hat{X}>x_{m, k_{m}}\right)$ where $k_{m}$ is a sequence such that $x_{m-1, k_{m}-1}<x \leq x_{m, k_{m}}$ for all $m$. We have the following convergence result.

Proposition $5\left(X_{i}^{*}, \ldots, X_{n}^{*}, Q\right)$ is a CCE in the original DU-comonotone market, where $X_{i}^{*}=f_{i}(X)-\mathbb{E}^{Q}\left[f_{i}(X)\right]+\mathbb{E}^{Q}\left[\xi_{i}\right]$ for $i \in N$.

Proof Consider $R(\hat{X})=\{k \varepsilon: k=1, \ldots, m\}$, initial wealth allocations $\psi_{0}^{i}(\hat{X})$ for $i \in$ $N$ and pricing measure $\hat{Q}_{0}$. In step (2-a), $r_{j-1}^{i}=Q_{i}\left(\hat{X} \geq x_{j}\right)=Q_{i}(X>(j-1) \varepsilon)$ and $r_{j-1}^{i}$ is independent with the choice of pricing measures for each $j=1, \ldots, m$. After the step (3) in the algorithm, we have

$$
\begin{aligned}
\psi_{m}^{i}(x) & =\sum_{k=1}^{m} \delta_{m, k}^{i} \mathbb{I}_{\left\{\hat{X}_{\left.\geq x_{k}\right\}}\right\}}(x), i \in N, \quad \text { and } \\
\hat{Q}_{m} & =\left(q_{m, k}\right)_{k=1, \ldots, m}
\end{aligned}
$$

where

$$
\begin{aligned}
& q_{m, k}=\frac{1}{2}\left(\operatorname{sl}_{i}\left\{r_{k-1}^{i}\right\}+\max _{i}\left\{r_{k-1}^{i}\right\}\right), \quad \text { and } \\
& \frac{\delta_{m, k}^{i}}{\varepsilon}= \begin{cases}1, & \text { if } r_{k-1}^{i}>q_{m, k}, \\
0, & \text { if } r_{k-1}^{i}<q_{m, k}, \\
\frac{\mathbb{I}_{\left\{\max _{i}\left\{r_{k-1}^{i}\right\}=q_{m, k}\right\}},}{\#\left\{r_{k-1}^{i}=q_{m, k}, i=1, \ldots, n\right\}}, & \text { if } r_{k-1}^{i}=q_{m, k} .\end{cases}
\end{aligned}
$$

Since $r_{j}^{i}, j=0, \ldots, m-1, i \in N$ are independent with the choice of pricing measures and the choice of initial allocation functions repeating the process (2)-(3) will lead to the same result as (36). Thus, we get the convergence result $\psi_{m}^{i}(x)$ and $\hat{Q}_{m}$ in (36) after the first round of step (2)-(3).

Let $\mathbb{Z}_{+}$be the set of positive integers. To show the convergence to a CCE, we take a sequence $\left\{\varepsilon_{m}=\frac{\bar{X}}{2^{m}}\right\}_{m \in \mathbb{Z}_{+}}$. For simplicity, we abuse notation slightly here: for each 
$m \in \mathbb{Z}_{+}$, on the discrete sample space $R\left(\hat{X}_{m}\right)=\left\{x_{m, k}=k \varepsilon_{m}=\frac{k \bar{X}}{2^{m}}, k=1, \ldots, 2^{m}\right\}$, the result obtained in the Algorithm is $\left(\left(\psi_{m}^{1}\left(\hat{X}_{m}\right), \ldots, \psi_{m}^{n}\left(\hat{X}_{m}\right)\right), \hat{Q}_{m}\right)$ where $\psi_{m}^{i}(x)=\sum_{k=1}^{2^{m}} \delta_{m, k}^{i} \mathbb{I}_{\left\{\hat{X}_{m} \geq x_{m, k}\right\}}(x), \hat{Q}_{m}=\left(q_{m, k}\right)_{k=1, \ldots, 2^{m}}, q_{m, k}=\frac{1}{2}\left(\operatorname{sl}_{i}\left\{r_{m, k-1}^{i}\right\}+\right.$ $\left.\max _{i}\left\{r_{m, k-1}^{i}\right\}\right)$ and $r_{m, k-1}^{i}=Q_{i}\left(\hat{X}_{m} \geq x_{m, k}\right)=Q_{i}\left(X>\frac{(k-1) \bar{X}}{2^{m}}\right)$.

Arbitrarily take $x_{0} \in(0, \bar{X})$, and take a sequence $k_{m}$ such that $x_{m, k_{m}-1}<x_{0} \leq$ $x_{m, k_{m}}$ for each $m \in \mathbb{Z}_{+}$. First Note that we have either $x_{m, k_{m}-1}=x_{m+1, k_{m+1}-1}$ or $x_{m+1, k_{m+1}-1}=\left(x_{m, k_{m}-1}+x_{m, k_{m}}\right) / 2$. It follows that $x_{m, k_{m}-1}$ increases to $x_{0}$, while $x_{m, k_{m}}$ decreases to $x_{0}$ as $m \rightarrow \infty$. Thus, $r_{m, k_{m}}^{i}=Q_{i}\left(X>x_{m, k_{m}}\right)$ increases in $m$ and $\lim _{m \rightarrow \infty} r_{m, k_{m}}^{i}=Q_{i}\left(X>x_{0}\right)$ for $i \in N$. Since

$$
\max _{i \neq j}\left\{\frac{1}{2}\left(r_{m, k_{m}}^{i}+r_{m, k_{m}}^{j}\right)\right\} \leq q_{m, k_{m}+1} \leq \max _{i}\left\{r_{m, k_{m}}^{i}\right\},
$$

passing both sides to the limit gives us

$$
\begin{aligned}
\operatorname{sl}_{i}\left\{Q_{i}\left(X>x_{0}\right)\right\} & \leq \frac{1}{2}\left(\operatorname{sl}_{i}\left\{Q_{i}\left(X>x_{0}\right)\right\}+\max _{i}\left\{Q_{i}\left(X>x_{0}\right)\right\}\right) \\
& \leq \lim _{m \rightarrow \infty} q_{m, k_{m}+1} \leq \max _{i}\left\{Q_{i}\left(X>x_{0}\right)\right\} .
\end{aligned}
$$

That is $\operatorname{sl}_{i}\left\{g_{i}\left(\mathbb{P}\left(X>x_{0}\right)\right)\right\} \leq Q\left(X>x_{0}\right) \leq \max _{i}\left\{g_{i}\left(\mathbb{P}\left(X>x_{0}\right)\right)\right\}$, i.e., condition (i) in Theorem 1 is satisfied. Denote $f_{i}(x)=\lim _{m \rightarrow \infty} \psi_{m}^{i}(x)$ for all $x \in[0, \bar{X}]$. Then,

$$
\begin{aligned}
f_{i}^{\prime}\left(x_{0}\right) & =\lim _{k \rightarrow \infty} 2^{k} \lim _{m \rightarrow \infty}\left(\psi_{m}^{i}\left(x_{0}+2^{-k}\right)-\psi_{m}^{i}\left(x_{0}\right)\right) \\
& =\lim _{k \rightarrow \infty} 2^{k} \lim _{k \leq m \rightarrow \infty}\left(\delta_{m, k_{m}+1}^{i}+\cdots+\delta_{m, k_{m}+2^{m-k}}^{i}\right)
\end{aligned}
$$

If $g_{i}\left(\mathbb{P}\left(X>x_{0}\right)\right)>Q\left(X>x_{0}\right)=\lim _{m \rightarrow \infty} q_{m, k_{m}+1}$, there exists $\Delta_{1}, \Delta_{2}>0$ such that $g_{i}(\mathbb{P}(X>x))>Q(X>x)+\Delta_{2}$ for all $x \in\left[x_{0}, x_{0}+\Delta_{1}\right)$. Consider cases $2^{-k}<\Delta_{2}$ only. For each $k$, when $m$ is large enough, we have $q_{m, k_{m}+l}<r_{m, k_{m}+l-1}^{i}$ and thus $\delta_{m, k_{m}+l}=2^{-m}$ for $l=1, \ldots, 2^{m-k}$. It follows that $f_{i}^{\prime}\left(x_{0}\right)=1$. If $g_{j}(\mathbb{P}(X>$ $\left.\left.x_{0}\right)\right)<Q\left(X>x_{0}\right)=\lim _{m \rightarrow \infty} q_{m, k_{m}+1}$, we can use similar argument as above to conclude that $\delta_{m, k_{m}+l}=0$ for $l=1, \ldots, 2^{m-k}$ and thus $f_{i}^{\prime}\left(x_{0}\right)=0$. Therefore, $X_{i}^{*}=f_{i}(X)-\mathbb{E}^{Q}\left[f_{i}(X)\right]+\mathbb{E}^{Q}\left[\xi_{i}\right], i \in N$ satisfy Condition (ii) in Theorem 1. According to Theorem $1,\left(\left(X_{i}^{*}, \ldots, X_{n}^{*}, Q\right)\right.$ is a CCE in the original DU-comonotone market.

\section{References}

Anthropelos, M., Kardaras, C.: Equilibrium in risk-sharing games. Finance Stoch. 21(3), 815-865 (2017) Araujo, A., Chateauneuf, A., Faro, J.H.: Pricing rules and Arrow-Debreu ambiguous valuation. Econ. Theory 49, 1-35 (2012). https://doi.org/10.1007/s00199-011-0660-4 
Arrow, K.J., Debreu, G.: Existence of an equilibrium for a competitive economy. Econometrica 22(3), 265-290 (1954)

Artzner, P., Delbaen, F., Eber, J.-M., Heath, D.: Coherent measures of risk. Math. Finance 9(3), 203-228 (1999)

Barrieu, P., El Karoui, N.: Inf-convolution of risk measures and optimal risk transfer. Finance Stoch. 9(2), 269-298 (2005)

Beissner, P., Riedel, F.: Equilibria under Knightian price uncertainty. Econometrica 87(1), 37-64 (2019)

Bernard, C., He, X., Yan, J.A., Zhou, X.Y.: Optimal insurance design under rank-dependent expected utility. Math. Finance 25, 154-186 (2015)

Bewley, T.F.: Existence of equilibria in economics with infinitely many commodities. J. Econ. Theory 4(3), 514-540 (1972)

Boonen, T.J.: Competitive equilibria with distortion risk measures. ASTIN Bull. 45(3), 703-728 (2015)

Boonen, T.J.: Risk redistribution games with dual utilities. ASTIN Bull. 47(1), 303-329 (2017)

Borch, K.: Equilibrium in a reinsurance market. Econometrica 30, 424-444 (1962)

Cai, J., Lemieux, C., Liu, F.: Optimal reinsurance from the perspectives of both an insurer and a reinsurer. ASTIN Bull. 46(3), 815-849 (2016)

Cai, J., Liu, H., Wang, R.: Pareto-optimal reinsurance arrangements under general model settings. Insur. Math. Econ. 77, 24-37 (2017)

Carlier, G., Dana, R.-A.: Pareto efficient insurance contracts when the insurer's cost function is discontinuous. Econ. Theory 21, 871-893 (2003). https://doi.org/10.1007/s00199-002-0281-z

Chabi-Yo, F.: Pricing kernels with stochastic skewness and volatility risk. Manag. Sci. 58(3), 624-640 (2012)

Chateauneuf, A., Dana, R.-A., Tallon, J.-M.: Optimal risk-sharing rules and equilibria with Choquetexpected-utility. J. Math. Econ. 34(2), 191-214 (2000)

Cheng, H.H.: Asset market equilibrium in infinite dimensional complete markets. J. Math. Econ. 20(1), 137-152 (1991)

Cheung, K.C., Sung, K., Yam, S.C.P.: Risk-minimizing reinsurance protection for multivariate risks. J. Risk Insur. 81(1), 219-236 (2014)

Chew, S.H., Karni, E., Safra, Z.: Risk aversion in the theory of expected utility with rank dependent probabilities. J. Econ. Theory 42, 370-381 (1987)

Dana, R.-A.: Existence and uniqueness of equilibria when preferences are additively separable. Econometrica 61(4), 953-957 (1993)

Dana, R.-A., Le Van, C.: Arbitrage, duality and asset equilibria. J. Math. Econ. 34(3), 397-413 (2000)

Dana, R.-A., Le Van, C.: Overlapping sets of priors and the existence of efficient allocations and equilibria for risk measures. Math. Finance 20(3), 327-339 (2010)

Dana, R.-A., Riedel, F.: Intertemporal equilibria with Knightian uncertainty. J. Econ. Theory 148, 15821605 (2013)

De Giorgi, E., Hens, T., Rieger, M.O.: Financial market equilibria with cumulative prospect theory. J. Math. Econ. 46(5), 633-651 (2010)

Delbaen, F.: Monetary Utility Functions. Osaka University Press, Osaka (2012)

Denneberg, D.: Non-additive Measure and Integral. Springer, Berlin (1994)

Denuit, M., Dhaene, J., Goovaerts, M., Kaas, R.: Actuarial Theory for Dependent Risks: Measures, Orders and Models. Wiley, New York (2005)

Dybvig, P.H.: Distributional analysis of portfolio choice. J. Bus. 61(3), 369-393 (1988)

Embrechts, P., Puccetti, G., Rüschendorf, L., Wang, R., Beleraj, A.: An academic response to Basel 3.5. Risks 2(1), 25-48 (2014)

Embrechts, P., Liu, H., Wang, R.: Quantile-based risk sharing. Oper. Res. 66(4), 936-949 (2018)

Embrechts, P., Liu, H., Mao, T., Wang, R.: Quantile-based risk sharing with heterogeneous beliefs. Math. Program. 181(2), 319-347 (2020)

Föllmer, H., Schied, A.: Stochastic Finance. An Introduction in Discrete Time, 4th edn. Walter de Gruyter, Berlin (2016)

Heath, D., Ku, H.: Pareto equilibria with coherent measures of risk. Math. Finance 14(2), 163-172 (2004)

Heaton, J., Lucas, D.J.: Evaluating the effects of incomplete markets on risk sharing and asset pricing. J. Polit. Econ. 104(3), 443-487 (1996)

Hens, T., Reichlin, C.: Three solutions to the pricing kernel puzzle. Rev. Financ. 17, 1065-1098 (2013)

Huberman, G., Mayers, D., Smith Jr., C.W.: Optimal insurance policy indemnity schedules. ACA Trans. 14(2), 415-426 (1983) 
Jarrow, R.: Heterogeneous expectations, restrictions on short sales, and equilibrium asset prices. J. Finance 35(5), 1105-1113 (1980)

Jin, H., Xia, J., Zhou, X.Y.: Arrow-Debreu equilibria for rank-dependent utilities with heterogeneous probability weighting. Math. Finance 29(3), 898-927 (2019)

Jouini, E., Schachermayer, W., Touzi, N.: Optimal risk sharing for law invariant monetary utility functions. Math. Finance 18(2), 269-292 (2008)

Landsberger, M., Meilijson, I.: Co-monotone allocations, Bickel-Lehmann dispersion and the Arrow-Pratt measure of risk aversion. Ann. Oper. Res. 52(2), 97-106 (1994)

Ludkovski, M., Rüschendorf, L.: On comonotonicity of Pareto optimal risk sharing. Stat. Probab. Lett. 78(10), 1181-1188 (2008)

Ludkovski, M., Young, V.R.: Optimal risk sharing under distorted probabilities. Math. Financ. Econ. 2(2), 87-105 (2009)

Mas-Colell, A., Whinston, M.D., Green, J.R.: Microeconomic Theory. Oxford University Press, New York (1995)

Moulin, H.: Egalitarian-equivalent cost sharing of a public good. Econometrica 55(4), 963-976 (1987)

Moulin, H., Shenker, S.: Serial cost sharing. Econometrica 60(5), 1009-1037 (1992)

Müller, A., Scarsini, M., Tsetlin, I., Winkler, R.L.: Between first and second-order stochastic dominance. Manag. Sci. 63(9), 2933-2947 (2017)

Nielsen, L.T.: Asset market equilibrium with short-selling. Rev. Econ. Stud. 56(3), 467-473 (1989)

Quiggin, J.: A theory of anticipated utility. J. Econ. Behav. Org. 3(4), 323-343 (1982)

Quiggin, J.: Generalized Expected Utility Theory: The Rank-Dependent Model. Kluwer, Dohdridge (1993)

Rieger, M.O., Wang, M.: Cumulative prospect theory and the St. Petersburg paradox. Econ. Theory 28, 665-679 (2006). https://doi.org/10.1007/s00199-005-0641-6

Rüschendorf, L.: Mathematical Risk Analysis: Dependence, Risk Bounds, Optimal Allocations and Portfolios. Springer, Heidelberg (2013)

Schmeidler, D.: Integral representation without additivity. Proc. Am. Math. Soc. 97(2), 255-261 (1986)

Schmidt, U., Zank, H.: Risk aversion in cumulative prospect theory. Manag. Sci. 54, 208-216 (2008)

Tsanakas, A., Christofides, N.: Risk exchange with distorted probabilities. ASTIN Bull. 36(1), 219-243 (2006)

Tversky, A., Kahneman, D.: Advances in prospect theory: cumulative representation of uncertainty. J. Risk Uncertain. 5(4), 297-323 (1992)

Wang, S.S., Young, V.R., Panjer, H.H.: Axiomatic characterization of insurance prices. Insur. Math. Econ. 21(2), 173-183 (1997)

Werner, J.: Arbitrage and the existence of competitive equilibrium. Econometrica 55(6), 1403-1418 (1987)

Wilson, R.: The theory of syndicates. Econometrica 36, 119-132 (1968)

Xia, J., Zhou, X.Y.: Arrow-Debreu equilibria for rank-dependent utilities. Math. Finance 26(3), 558-588 (2016)

Xu, Z.Q., Zhou, X.Y., Zhuang, S.C.: Optimal insurance under rank-dependent utility and incentive compatibility. Math. Finance 29(2), 659-692 (2019)

Yaari, M.E.: The dual theory of choice under risk. Econometrica 55(1), 95-115 (1987)

Publisher's Note Springer Nature remains neutral with regard to jurisdictional claims in published maps and institutional affiliations. 